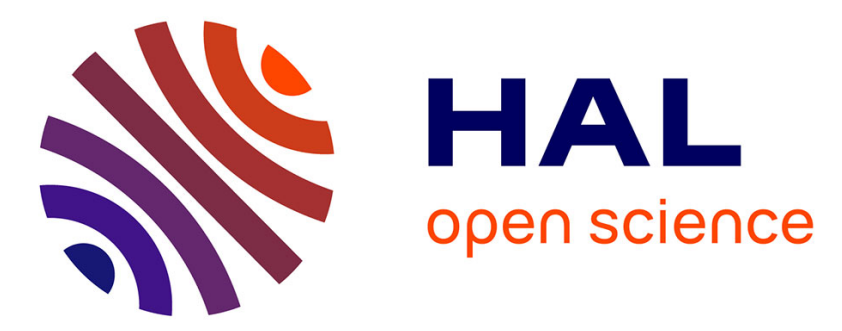

\title{
Time-response shaping using Output to Input Saturation Transformation
}

E. Chambon, L. Burlion, P. Apkarian

\section{To cite this version:}

E. Chambon, L. Burlion, P. Apkarian. Time-response shaping using Output to Input Saturation Transformation. International Journal of Control, 2017, 90, pp.1 - 32. 10.1080/00207179.2017.1286043 . hal-01633711

\section{HAL Id: hal-01633711 https://hal.science/hal-01633711}

Submitted on 15 Nov 2017

HAL is a multi-disciplinary open access archive for the deposit and dissemination of scientific research documents, whether they are published or not. The documents may come from teaching and research institutions in France or abroad, or from public or private research centers.
L'archive ouverte pluridisciplinaire HAL, est destinée au dépôt et à la diffusion de documents scientifiques de niveau recherche, publiés ou non, émanant des établissements d'enseignement et de recherche français ou étrangers, des laboratoires publics ou privés. 
Submitted to the International Journal of Control

Vol. 00, No. 00, Month 20XX, 1-32

\title{
Time-response shaping using Output to Input Saturation Transformation
}

\author{
E. Chambon ${ }^{\mathrm{a} *}$, L. Burlion ${ }^{\mathrm{a}}$ and P. Apkarian ${ }^{\mathrm{a}}$ \\ ${ }^{a}$ Onera - The French Aerospace Lab, 2 Avenue Édouard Belin, FR-31055, Toulouse, France
}

(Received 00 Month 20XX; accepted 00 Month 20XX)

\begin{abstract}
For linear systems, the control law design is often performed so that the resulting closed-loop meets specific frequency-domain requirements. However, in many cases, it may be observed that the obtained controller does not enforce time-domain requirements amongst which the objective of keeping an output variable in a given interval. In this article, a transformation is proposed to convert prescribed bounds on an output variable into time-varying saturations on the synthesized linear control law. This transformation uses some well-chosen time-varying coefficients so that the resulting time-varying saturations do not overlap in the presence of disturbances. Using an anti-windup approach, it is demonstrated that the origin of the resulting closed-loop is globally asymptotically stable and that the regulated variable satisfies the time-domain constraints in the presence of an unknown finite-energy bounded disturbance. An application to a linear ball and beam model is presented.
\end{abstract}

Keywords: constrained control; linear systems; unknown disturbance; interval constraint; time-domain constraint; anti-windup

\section{Introduction}

To stabilise a given system, many techniques exist to obtain a control law satisfying to specified constraints. As far as MIMO systems are concerned, $H_{\infty}$ loop-shaping can for example be used to enforce frequency-domain requirements. However it is possible that, using such control law, time-domain requirements on a so-called regulated variable $\alpha=\mathbf{C}_{\alpha} \boldsymbol{y} \in \mathbb{R}$ are not fulfilled. This is illustrated on Fig. 1 where $\alpha$ time-response violates expected bounds $[\underline{\alpha}(t), \bar{\alpha}(t)]$. In practice, a good knowledge of the studied system is often sufficient to shape its time-response. However, designing controllers satisfying to such prescribed time-domain requirements remains tedious and relies on numerous trial-and-errors involving simulations. Consequently, for more complex systems, and from the theoretical point of view, dedicated methods are often required to enforce both stability and time-domain constraints.

Amongst existing strategies to enforce time-domain requirements like response-time or overshoot limitation, it is possible to mention the work presented in Gevers (2002) which introduced the notion of Iterative Feedback Tuning (IFT). The idea is to shape the closed-loop in response to specific input signals so as to satisfy time-domain constraints. In the PID-tuning case, a comparison with practitioners methods was performed in Mossberg, Gevers, and Lequin (2002) which gives a hint on how to achieve time-domain requirements using this method. Time-domain specifications are also treated through optimal control strategies as extensively presented in Goodwin, Seron, and de Doná (2005). These approaches include model predictive control (MPC) in which the optimisation problem can take constraints into account, see for example Chen and Allgöwer (1996) or Chen and Allgöwer (1999). Computationally effective methods close to MPC are reported in Ghaemi, Sun, and Kolmanovsky (2012). The notion of reference-governor to adjust the reference trajectory so that the constraints on the system are satisfied is also noticeable. It was presented in Gilbert and

*Corresponding author. Email: emmanuel.chambon@onera.fr 
Kolmanovsky (2002) with an application to aerospace systems in Polóni, Kalabić, McDonough, and Kolmanovsky (2014). The combination of frequency-domain and time-domain constraints has been explored in Apkarian, Ravanbod-Hosseini, and Noll (2011) and references therein. This method makes use of non-smooth bundle optimization methods and is referred to as "constrained structured $\mathrm{H}_{\infty}$-synthesis". It combines simulation optimization with $\mathrm{H}_{\infty}$ synthesis to enforce both frequencyand time-domain requirements.

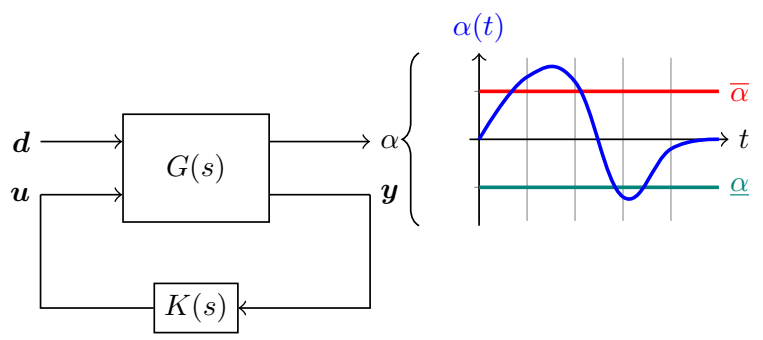

Figure 1. Illustration of system $(G)$ in closed-loop with controller $K$. The requirement on the regulated variable $\alpha(t)$ is violated.

These strategies often include the control law design, especially when an optimising scheme is used. Alternative schemes including anti-windup systems were proposed for example in Turner and Postlethwaite (2002) and Rojas and Goodwin (2002). Compared to the aforementioned results, the anti-windup design is interesting because the nominal control law remains unchanged when acting far from the constraints. Also, an extensive literature on the subject is available, see Tarbouriech and Turner (2009) or Galeani, Tarbouriech, Turner, and Zaccarian (2009) for instance. However, there is not necessarily a guarantee on the fact that the time-domain constraints will actually be satisfied. In this article, the approach presented in Burlion (2012) and applied for example in Burlion and de Plinval (2013) is presented in-depth for state-feedback minimum-phase linear systems subject to disturbances. The output- to input-saturation transformation (OIST) theory proposes to reformulate prescribed bounds on the regulated variable $\alpha$ into state-dependent saturations on the control input $u$. This approach is illustrated on Fig. 2 where an ad hoc saturating block is inserted before the system control input. As indicated in this figure, additional information may be required to express these saturations. Using this method along with some assumptions, it is possible to obtain guarantees on the fulfilment of the time-domain constraints when an unsatisfactory control law has already been designed.

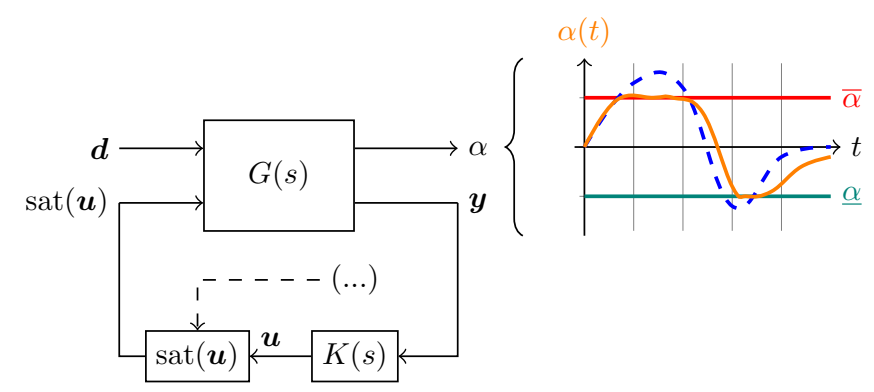

Figure 2. Illustration of system $(G)$ in closed-loop with the saturations obtained using OIST, and expected performance result.

This article is an extension of the work presented in Chambon, Burlion, and Apkarian (2015a). It gives a comprehensive description of the method for minimum-phase linear systems with unknown finite-energy bounded disturbances. It also provides a stability proof when inserting the obtained saturations in the closed-loop. Other cases of application were presented in Chambon, Burlion, and Apkarian (2015b).

The article is organised as follows: the ball and beam example is introduced in Sect. 2 as a 
case study to introduce the OIST problem formulation. Notations and definitions along with some functions properties are also presented in this section as well as the assumptions satisfied by the considered class of systems. Then, the output to input saturations transformation proposed to solve this problem is presented in Sect. 3. Due to the conservatism introduced by the bounds on the disturbances, special attention is paid in the selection of the design parameters so that the resulting time-varying saturations do not overlap ${ }^{1}$. Stability of the system in closed-loop with the obtained saturated control is established in Sect. 4. Finally, the whole approach is applied to the linear ball and beam model in Sect. 5. Conclusions and perspectives are then presented in Sect. 6 .

\section{Problem formulation}

\subsection{Definitions and notations}

Before introducing the problem in Sect. 2 and for the sake of consistency, some definitions and notations are presented or recalled here.

\subsubsection{Acronyms}

The acronyms listed in Tab. 1 are used throughout the article.

\begin{tabular}{ll} 
Table 1. & Acronyms. \\
\hline Acronym & Definition \\
\hline CICS & Converging Input Converging State \\
GAS & Globally Asymptotically Stable (system) \\
GES & Globally Exponentially Stable (system) \\
ISS & Input-to-state Stable (system) \\
LQR & Linear-Quadratic Regulator \\
LTI & Linear Time-Invariant \\
MIMO & Multiple Inputs Multiple Outputs (system) \\
OIST & Output to Input Saturation Transformation \\
\hline
\end{tabular}

\subsubsection{Notations}

If not stated otherwise, the state-space representation of a LTI system $(G)$ is denoted $G=$ $(\mathbf{A}, \mathbf{B}, \mathbf{C}, \mathbf{D})$ where $\mathbf{A} \in \mathbb{R}^{n \times n}, \mathbf{B}=\left[\begin{array}{lll}\mathbf{B}_{\mathbf{d}} & \mathbf{B}_{\mathbf{u}} & \mathbf{B}_{\mathbf{e}}\end{array}\right] \in \mathbb{R}^{n \times l}, \mathbf{C} \in \mathbb{R}^{m \times n}$ and $\mathbf{D}=\left[\begin{array}{lll}\mathbf{D}_{\mathbf{d}} & \mathbf{D}_{\mathbf{u}} & \mathbf{D}_{\mathbf{e}}\end{array}\right] \in$ $\mathbb{R}^{m \times l}$. The state vector is then denoted $\boldsymbol{x} \in \mathbb{R}^{n}$ and the measurements vector is denoted $\boldsymbol{y} \in \mathbb{R}^{m}$ with $m \leq n$. The input vector lying in $\mathbb{R}^{l}$ is divided between the control inputs denoted $\boldsymbol{u}$ and the unknown inputs (disturbances) which are denoted $\boldsymbol{d}$ (state disturbance) and $\boldsymbol{e}$ (measurements disturbance). The transfer function from an input $\boldsymbol{u}$ to an output $\boldsymbol{y}$ is denoted $T_{\boldsymbol{u} \rightarrow \boldsymbol{y}}(s)$ where $s$ is the Laplace variable.

Inequalities involving matrices of identical dimensions are understood component-wise: let $(\mathbf{A}, \mathbf{B}) \in \mathbb{R}^{n \times m}$, then, $\mathbf{A} \leq \mathbf{B} \Leftrightarrow \forall 1 \leq i \leq n, \forall 1 \leq j \leq m, A_{i j} \leq B_{i j}$.

For a given bounded vector $\boldsymbol{x}(t)$, if the bounds are known they are denoted $(\underline{\boldsymbol{x}}(t), \overline{\boldsymbol{x}}(t))$, i.e. $\forall t, \underline{\boldsymbol{x}}(t) \leq \boldsymbol{x}(t) \leq \overline{\boldsymbol{x}}(t)$.

The saturation and deadzone functions applied to a bounded variable $\boldsymbol{x}$ are respectively denoted sat $\underline{\boldsymbol{x}}(\boldsymbol{x})$ and $\mathrm{Dz}_{\underline{\boldsymbol{x}}}^{\overline{\bar{x}}}(\boldsymbol{x})$. They are related to each other by

$$
\operatorname{sat}_{\underline{\boldsymbol{x}}}^{\overline{\bar{x}}}(\boldsymbol{x})=\boldsymbol{x}-\mathrm{Dz}_{\underline{\boldsymbol{x}}}^{\overline{\bar{x}}}(\boldsymbol{x})
$$

\footnotetext{
${ }^{1}$ For more details on how the notion is used in the following, the reader should refer to Def. 1.
} 
The standard Euclidean norm of a given signal $\boldsymbol{x}(t)$ defined for $t \geq 0$ is denoted $\|\boldsymbol{x}\|$. The $L_{2}$-norm of the same signal is denoted $\|\boldsymbol{x}\|_{2}$ and is given by

$$
\|\boldsymbol{x}\|_{2}=\sqrt{\int_{0}^{\infty}|\boldsymbol{x}(t)|^{2} \mathrm{~d} t}
$$

\subsubsection{Definitions}

Some definitions are now introduced, starting with the notion of the "overlap" of two signals:

Definition 1: In the article, the term "overlap" is used to refer to two signals taking the same value at a given instant and possibly changing order. Given two unidimensional signals $\underline{s}(t)$ and $\bar{s}(t)$, a definition of overlapping would thus be: $\exists t_{1}>0, \delta>0$ such that $\forall t<t_{1}, \underline{s}(t) \leq \bar{s}(t)$ and $\forall t \in\left[t_{1}, t_{1}+\delta[, \underline{s}(t)>\bar{s}(t)\right.$.

The Lambert function will be used to define constants. It is defined as follows:

Definition 2: Let $\forall x \in \mathbb{R}, F(x)=x \mathrm{e}^{x}$. The inverse function of $F$ is the Lambert function denoted $W_{0}(y)$ which fulfils $\forall y, F\left(W_{0}(y)\right)=W_{0}(y) \mathrm{e}^{W_{0}(y)}=y$.

This definition is used to define constants which in turn will help define differentiable approximates of some non-differentiable functions:

Definition 3: Let define the constants $\xi:=\frac{1}{2} W_{0}\left(\frac{1}{\mathrm{e}}\right)+\frac{1}{2}$ and $\Xi:=\xi-\tanh (\xi) \xi>0$. Using these notations, let also define the following functions, $\forall(x, y) \in \mathbb{R}^{2}$ :

$$
\begin{array}{ll}
f_{\text {abs }}(x) & :=\tanh (x) x+\Xi \\
f_{\max }(x, y) & :=\frac{1}{2}\left[x+y+f_{\text {abs }}(x-y)\right] \\
g(x, y) & :=f_{\max }\left(f_{\text {abs }}(x), f_{\text {abs }}(y)\right)
\end{array}
$$

which definition is extended to vectors $(\boldsymbol{x}, \boldsymbol{y}) \in \mathbb{R}^{n \times 2}$ in a component-wise manner. This definition triggers some remarks:

Remark 1: Note that $\xi$ is the solution to the equation $(2 x-1) \mathrm{e}^{2 x}=1$. Also note that $f_{\mathrm{abs}}, f_{\max }$ and $g$ are continuous differentiable over $\mathbb{R}$ or $\mathbb{R}^{2}$. Moreover,

- $\forall x \in \mathbb{R}, f_{\text {abs }}(x) \geq|x|$;

- $\forall(x, y) \in \mathbb{R}^{2}, f_{\max }(x, y) \geq \max (x, y)$.

Proof. First, let $f(x)=(2 x-1) \mathrm{e}^{2 x}$. Then, $f(\xi)=W_{0}\left(\frac{1}{\mathrm{e}}\right) \mathrm{e}^{W_{0}\left(\frac{1}{\mathrm{e}}\right)} \mathrm{e}=\frac{1}{\mathrm{e}} \times \mathrm{e}=1$ by definition of the Lambert function. Second, using the basics of functional analysis on the function $h(x)$ defined by $\forall x, h(x)=f_{\text {abs }}(x)-|x|$, the inequalities in Remark 1 are achieved. As far as the function $f_{\max }$ is concerned, the same study is performed using the following equality: $\forall(x, y), \max (x, y)=$ $\frac{1}{2}[x+y+|x-y|]$.

The following definitions are directly related to the implementation of the OIST method.

Definition 4: Let $k \in \mathbb{N}$. Considering a LTI system $(G)$, the regulated output variable $\alpha(t)=$ $\mathbf{C}_{\alpha} \boldsymbol{y}(t)$ is said to be of relative degree $k$ with respect to $\boldsymbol{u}$ if and only if 


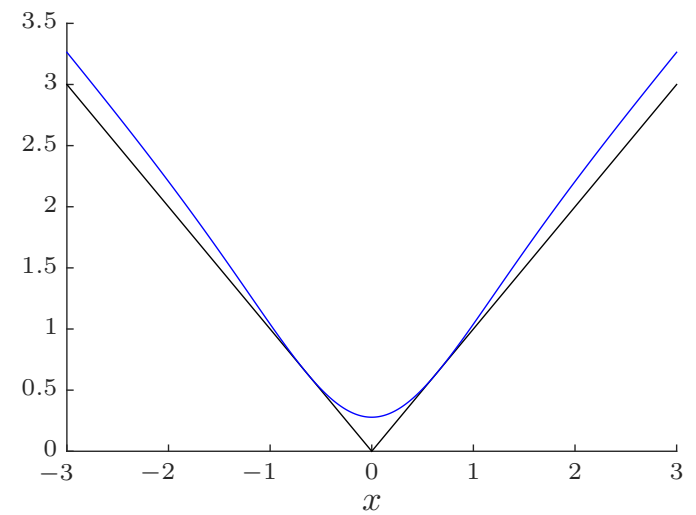

Figure 3. Representation of the absolute value function (in black) and its differentiable approximate $f_{\text {abs }}$ (in blue) over the interval $[-3,3]$.

$$
\forall 0 \leq i<k-1, \mathbf{C}_{\alpha} \mathbf{A}^{i} \mathbf{B}_{u}=0 \text { and } \mathbf{C}_{\alpha} \mathbf{A}^{k-1} \mathbf{B}_{u} \neq 0
$$

Definition 5: Let $S=\left[\begin{array}{lll}s_{0} & \ldots & s_{k}\end{array}\right] \in \mathbb{R}^{k+1}$. The function

$$
\sigma(S)=\left[\begin{array}{lll}
\sigma\left(s_{0}\right) & \ldots & \sigma\left(s_{k}\right)
\end{array}\right]
$$

where

$$
\sigma\left(s_{i}\right):=\left\{\begin{array}{lll}
s_{i-1} & \text { if } \quad 1 \leq i \leq k \\
s_{k} & \text { if } \quad i=0
\end{array}\right.
$$

is called the cyclic permutation of length $k+1$ on the elements of $S$.

\subsection{Case study}

In this section, an example is introduced where the control synthesis problem has been solved. In this case, there is a violation of the expected time-domain performance of the considered regulated variable $\alpha$ which motivates the formulation of a new problem in Sect. 2.3. The solution to this problem is the main result proposed in this article.

Note that in this example, a more thorough control design study may be sufficient to enforce the time-domain requirement. However, this is not considered in this article for two reasons:

- the OIST method was proposed to enforce time-domain requirements when the controller is not able to do so;

- other criteria often enter in the control design. Enforcing the time-domain criterion may degrade nominal performance from other points of view.

The OIST method proposes to achieve a time-domain requirement while ensuring the nominal ${ }^{2}$ performance. This case study is dedicated to the position control of a ball on a beam. The physical system and the notations are represented in Fig. 4.

${ }^{2}$ In the sense: when the time-domain condition is met. 


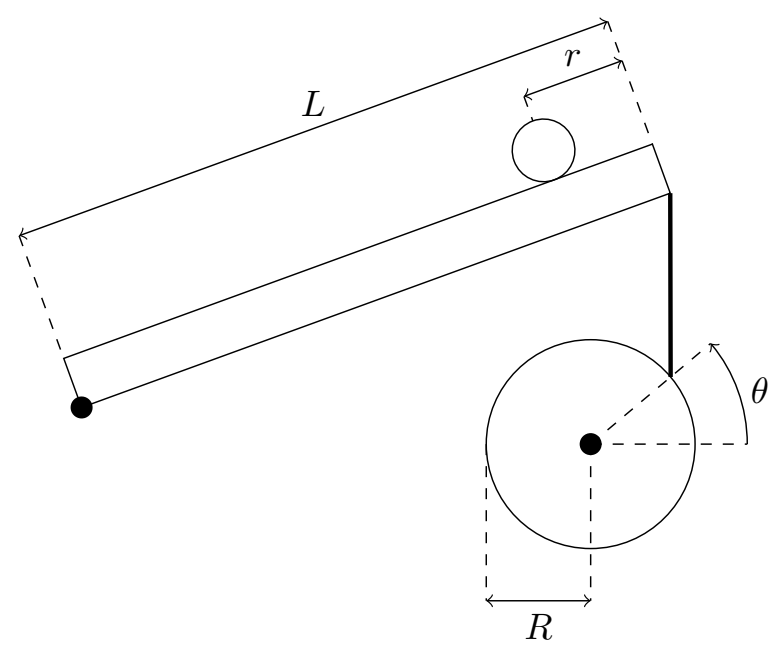

Figure 4. Ball and beam system example, thick dots represent fixed axes of rotation
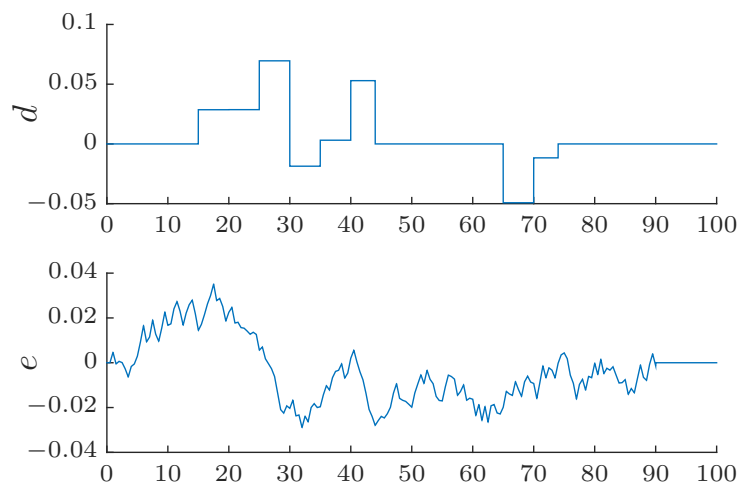

Figure 5. Disturbances $d$ and $e$ applied to the ball and beam system in simulation.

The beam is actuated using a lever arm. An unknown disturbance force $d$ is eventually applied to the ball acceleration and an unknown $\operatorname{bias}^{3} e$ is introduced on the measure of the ball position $r$. Both disturbances are represented on Fig. 5. The state vector of the system is defined by $\boldsymbol{x}=\left[\begin{array}{ll}r & \dot{r}\end{array}\right]^{\top}$ and the measurements vector by $\boldsymbol{y}=\boldsymbol{x}+\mathbf{D}_{e} e$ where $\mathbf{D}_{e}=\left[\begin{array}{ll}1 & 0\end{array}\right]^{\top}$. The regulated variable is defined as:

$$
\alpha(t)=\mathbf{C}_{\alpha} \boldsymbol{y}(t)=\mathbf{C}_{\alpha} \boldsymbol{x}(t)+\mathbf{C}_{\alpha} \mathbf{D}_{e} e(t)=\mathbf{C}_{\alpha} \boldsymbol{x}(t)+E_{\alpha} e(t)
$$

with $\mathbf{C}_{\alpha}=\left[\begin{array}{ll}1 & 0\end{array}\right]$ and $E_{\alpha}=1$. It corresponds to the measured disturbed ball position.

The reason for monitoring this variable is quite obvious. The beam length is limited to $L=1 \mathrm{~m}$ which means that even a theoretically stabilizing control law can result in the ball falling off the beam. The objective - i.e. the time-domain requirement - is thus to satisfy $\forall t, 0.1 \leq \alpha(t) \leq 0.9$ (in meters) while driving the system from $r_{0}=0.5 \mathrm{~m}$ to the setpoint $r_{s}=0.6 \mathrm{~m}$. The system state-space representation is given by:

\footnotetext{
${ }^{3}$ In simulation, a uniform number generator with limited rate is used
} 


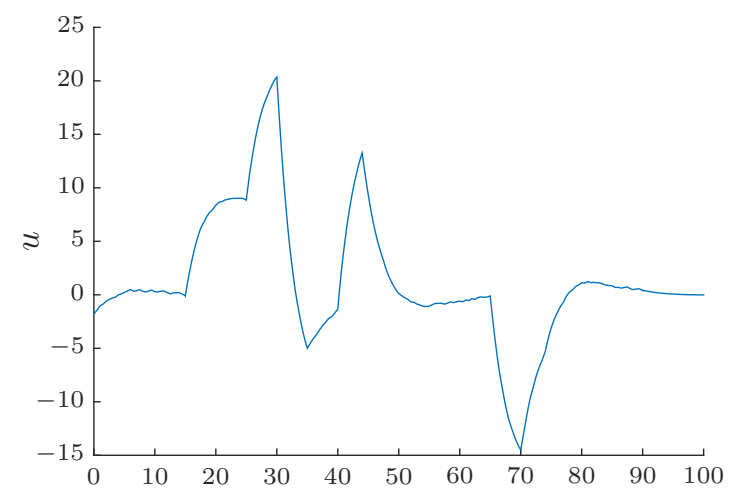

Figure 6. Simulation of the control signal $u$ using the controller with integral action defined in Eq. (10).

$$
\mathbf{A}=\left[\begin{array}{ll}
0 & 1 \\
0 & 0
\end{array}\right], \mathbf{B}=\left[\begin{array}{rrr}
0 & 0 & 0 \\
1 & -0.21 & 0
\end{array}\right], \mathbf{C}=I_{2}, \mathbf{D}=\left[\begin{array}{lll}
0 & 0 & 1 \\
0 & 0 & 0
\end{array}\right]
$$

where the inputs are respectively $d, u$ and $e$. As far as the nominal control design is concerned, it is decided to implement a state-feedback controller with integral action to achieve steady-state accuracy. Let consider the following augmented system

$$
\mathbf{A}_{a}=\left[\begin{array}{cc}
\mathbf{A} & 0 \\
-\mathbf{C}_{\text {int }} & 0
\end{array}\right], \mathbf{B}_{a}=\left[\begin{array}{cc}
\mathbf{B}_{u} & 0 \\
0 & 1
\end{array}\right]
$$

where $\mathbf{B}_{u}=\left[\begin{array}{ll}0 & -0.21\end{array}\right]^{\top}$ and $\mathbf{C}_{\mathrm{int}} \boldsymbol{x}=\dot{r}$. Using LQR design on this augmented system with $\mathbf{R}=\operatorname{diag}\left(\left[\begin{array}{ll}10 & 4\end{array}\right]\right)$ and $\mathbf{Q}=\operatorname{diag}\left(\left[\begin{array}{lll}1 & 10 & 10\end{array}\right]\right)$, the following state-feedback gain $\mathbf{K}_{p}$ and integral action gain $\mathbf{K}_{i}$ are obtained:

$$
\mathbf{K}_{p}=\left[\begin{array}{rr}
-0.3156 & -2.0937 \\
0.0322 & -0.7661
\end{array}\right], \mathbf{K}_{i}=\left[\begin{array}{l}
0.0644 \\
1.5779
\end{array}\right]
$$

which state-space representation is given by

$$
(K)\left\{\begin{array}{l}
\dot{x}_{K}(t)=-\mathbf{K}_{i}(2) x_{K}(t)+\left[\begin{array}{ll}
0 & 1
\end{array}\right]\left\{\boldsymbol{y}_{s}-\boldsymbol{y}(t)\right\} \\
\boldsymbol{y}_{K}(t)=-\mathbf{K}_{i} x_{K}(t)+\mathbf{K}_{p}\left\{\boldsymbol{y}_{s}-\boldsymbol{y}(t)\right\}
\end{array}\right.
$$

where $\boldsymbol{y}_{s}=\left[\begin{array}{ll}r_{s} & 0\end{array}\right]^{\top}$. This dynamic controller is stable and yields good results on $r_{s}$-setpoint tracking. The control signal simulation is shown on Fig. 6. However, violation of the time-domain requirement $0.1 \leq \alpha(t) \leq 0.9$ occurs as one can see on Fig. 7 .

\subsection{Problem formulation}

Let consider a LTI system $(G)$ with the following state-space representation: 


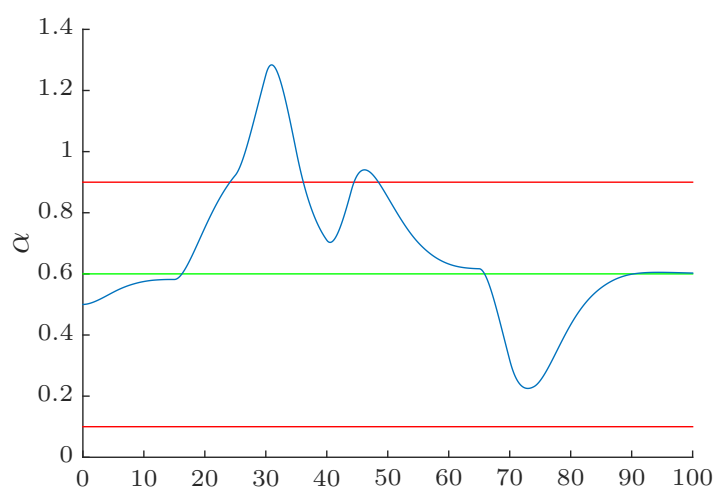

Figure 7. Simulation of the regulated variable $\alpha$ (in blue). The time-domain requirement (in red) is clearly violated. The set-point $r_{s}$ is represented in green.

$$
(G)\left\{\begin{aligned}
\dot{\boldsymbol{x}}(t) & =\mathbf{A} \boldsymbol{x}(t)+\mathbf{B}_{u} u(t)+\mathbf{B}_{d} d(t) & & \text { State } \\
\boldsymbol{y}(t) & =\boldsymbol{x}(t)+\mathbf{D}_{e} e(t) & & \text { Measurements } \\
\alpha(t) & =\mathbf{C}_{\alpha} \boldsymbol{x}(t)+\mathbf{E}_{\alpha} e(t) & & \text { Regulated variable }
\end{aligned}\right.
$$

where $u \in \mathbb{R}$ is the control input, $d \in \mathbb{R}$ is an unknown disturbance and $e \in \mathbb{R}$ is an unknown signal. The time-domain constraint consists in ensuring $\alpha(t) \in[\underline{\alpha}(t), \bar{\alpha}(t)], \forall t$ where $\underline{\alpha}(t)$ and $\bar{\alpha}(t)$ are design parameters. This is expressed in the following problem - which was introduced in Burlion (2012) and translated in the linear framework in Chambon et al. (2015a):

Problem 1: Find $[\underline{u}(t), \bar{u}(t)]$ and $\mathcal{C}_{0}$ such that

$$
\alpha(t) \in[\underline{\alpha}(t), \bar{\alpha}(t)], \forall t
$$

for the system described as

$$
\left\{\begin{aligned}
\dot{\boldsymbol{x}}(t) & =\mathbf{A} \boldsymbol{x}(t)+\mathbf{B}_{u} u(t)+\mathbf{B}_{d} d(t) \\
\boldsymbol{y}(t) & =\boldsymbol{x}(t)+\mathbf{D}_{e} e(t) \\
\alpha(t) & =\mathbf{C}_{\alpha} \boldsymbol{x}(t)+E_{\alpha} e(t) \\
u(t) & \in[u(t), \bar{u}(t)] \\
\boldsymbol{x}_{0} & \in \mathcal{C}_{0}
\end{aligned}\right.
$$

where $\boldsymbol{x} \in \mathbb{R}^{n}, \boldsymbol{y} \in \mathbb{R}^{m}, u \in \mathbb{R}$ is the command input, $d \in \mathbb{R}$ is an unknown disturbance and $e \in \mathbb{R}$ is an unknown signal with $E_{\alpha} \in \mathbb{R}$.

Remark 2: Note that $d$ and $e$ are chosen as unidimensional signals. This is non-limiting as long as the hypotheses are satisfied on each component in case of multidimensional signals.

Finding a solution to this problem motivates the Output to Input Saturation Transformation (OIST) where a constraint on the output $\alpha$ is transformed into saturations $[\underline{u}(t), \bar{u}(t)]$ on the control input $u$. 


\subsection{Assumptions}

Prior to the application of the transformation which will be presented in Sect. 3, the considered system has to fulfil some assumptions which are recalled here. First, the time-domain requirements needs to be meaningful:

Assumption 1: It is assumed that the expected bounds on the regulated variable $\alpha$ are chosen consistently:

$$
\forall t, \bar{\alpha}(t) \geq \underline{\alpha}(t)
$$

Assumption 2: The time-domain requirement signals are supposed to converge towards constant values satisfying Assum. 1:

$$
\lim _{t \rightarrow \infty} \underline{\alpha}(t)=\underline{\alpha}^{\star}, \lim _{t \rightarrow \infty} \bar{\alpha}(t)=\bar{\alpha}^{\star}
$$

Second, the relative degrees of the regulated variable with respect to the inputs is detailed:

Assumption 3: Let $(k, l) \in \mathbb{N}^{2}$ such that $1 \leq l \leq k$. It is supposed the regulated variable $\alpha$ is of relative degree $k$ (resp. l) with respect to $u$ (resp. $d$ ).

Let $\mathcal{D}(t)=\left[\begin{array}{llll}d & \dot{d} & \ldots & d^{(k-l-1)}\end{array}\right]^{\top} \in \mathbb{R}^{k-l}$ and $\mathcal{E}(t)=\left[\begin{array}{llll}e & \dot{e} & \ldots & e^{(k)}\end{array}\right]^{\top} \in \mathbb{R}^{k+1}$. The next assumption makes sure that these quantities are bounded by known time-varying matrices.

Assumption 4: Continuous time-varying bounds $[\underline{\mathcal{D}}(t), \overline{\mathcal{D}}(t)]$ on the unknown disturbance and its derivatives $\mathcal{D}(t)$ are supposed to be known, that is

$$
\underline{\mathcal{D}}(t) \leq \mathcal{D}(t) \leq \overline{\mathcal{D}}(t), \forall t
$$

or, more precisely,

$$
\forall 0 \leq i \leq k-l-1, \underline{d^{(i)}}(t) \leq d^{(i)}(t) \leq \overline{d^{(i)}}(t), \forall t
$$

The same holds for $\mathcal{E}(t)$ with time-varying bounds $[\underline{\mathcal{E}}(t), \overline{\mathcal{E}}(t)]$. Also:

$$
\lim _{t \rightarrow \infty} \underline{\mathcal{D}}(t)=\underline{\mathcal{D}}^{\star}, \lim _{t \rightarrow \infty} \overline{\mathcal{D}}(t)=\overline{\mathcal{D}}^{\star}, \lim _{t \rightarrow \infty} \underline{\mathcal{E}}(t)=\underline{\mathcal{E}}^{\star} \text { and } \lim _{t \rightarrow \infty} \overline{\mathcal{E}}(t)=\overline{\mathcal{E}}^{\star}
$$

where $\underline{\mathcal{D}}^{\star}=\left[\begin{array}{llll}\underline{d}^{\star} & 0 & \ldots & 0\end{array}\right]$, etc.

As conservative as this assumption may be, it is not so different from supposing the disturbances follow some theoretical model. The following assumptions will be used in the proof of the system state convergence in closed-loop:

Assumption 5: The disturbance $d$ is supposed to be of finite energy $\|d\|_{2}<\infty$. The same holds for e with energy $\|e\|_{2}<\infty$.

Assumption 6: Let $K(s)=\left(\mathbf{A}_{K}, \mathbf{B}_{K}, \mathbf{C}_{K}, \mathbf{D}_{K}\right)$ a strictly stable controller with state vector dimension $n_{K}$. It is supposed the control law $u=K(s) \boldsymbol{y}$ asymptotically (resp. exponentially) stabilizes 
system $(G)$ to the origin $\boldsymbol{x}^{\star}=0$ (without loss of generality), under Assum. 5 (resp. $d=0$ and $e=0)$. The controller state at the equilibrium is denoted $\boldsymbol{x}_{K}^{\star}=0$.

In this article, only minimum-phase systems are considered. In the non-minimum-phase case, additional analysis is required to ensure stability, which is considered as a perspective for future works.

Assumption 7: The zeros of the transfer function $T_{u \rightarrow \alpha}(s)$ from the control input $u$ to the regulated variable $\alpha$ are supposed to be of strictly negative real part.

To simplify the formulation of iterative expressions for the saturations components, the following assumption is made to ensure some terms will not re-appear upon derivation of the components:

Assumption 8: The relative degrees in Assum. 3 satisfy to the relation $2 l>k$.

For any system fulfilling these hypotheses, the Output to Input Saturation Transformation (OIST) is proposed as a solution to $\mathrm{Pb}$. 1. This transformation is detailed in the following section while closed-loop stability is established in Sect. 4.

\section{Output to Input Saturation Transformation}

The Output to Input Saturation Transformation is presented in this section in the case of known LTI systems fulfilling Assum. 3 to 8. In presence of unknown disturbances $d$ and $e$, time-varying coefficients are used in the obtained saturations so as to avoid overlap. Their expressions are derived from the known bounds on $\mathcal{D}(t)$ and $\mathcal{E}(t)$.

\subsection{Regulated variable differentiation and relative degree}

Using Assum. 3 and 8, the $k$-th derivative of the regulated variable $\alpha$ in function of $u, d$ and $e^{(k)}$ has the following expression:

$$
\alpha^{(k)}(t)=\mathbf{C}_{\alpha} \mathbf{A}^{k} x(t)+E_{\alpha} e^{(k)}(t)+\mathbf{C}_{\alpha} \mathbf{A}^{k-1} \mathbf{B}_{u} u(t)+\sum_{j=l}^{k} \mathbf{C}_{\alpha} \mathbf{A}^{j-1} \mathbf{B}_{d} d^{(k-j)}(t)
$$

By definition of the relative degree (Def. 4), the $k$-th derivative of the regulated variable $\alpha$ thus depends on the control input signal $u(t)$. In the next section, a lemma will be formulated so that properly differentiated design bounds on $\alpha$ can lead to saturations on $u$, using Eq. (20).

\section{$3.2 \quad$ Fulfilling the time-domain requirement}

Considering Pb. 1 , the objective is to ensure $\alpha(t) \in[\underline{\alpha}(t), \bar{\alpha}(t)], \forall t$. In this section, it is shown how adequate constraints on the successive derivatives of $\alpha$ can be used to fulfil this requirement. Let consider a vector of known positive time-varying signals

$$
\boldsymbol{\kappa}(t)=\left[\begin{array}{lll}
\kappa_{1}(t) & \ldots & \kappa_{k}(t)
\end{array}\right] \in \mathbb{R}_{+}^{k}
$$

which will act as "design parameters". Let $\underline{\alpha}^{(0)}(t)=\underline{\alpha}(t)$ and $\overline{\alpha^{(0)}}(t)=\bar{\alpha}(t)$. Also consider the vectors $\mathcal{A}(t)=\left[\begin{array}{llll}\alpha(t) & \ldots & \alpha^{(k)}(t)\end{array}\right]^{\top}, \underline{\boldsymbol{\Omega}}(t)=\left[\begin{array}{lll}\underline{\alpha^{(0)}}(t) & \ldots & \underline{\alpha^{(k)}}(t)\end{array}\right]^{\top}$ and $\overline{\boldsymbol{\Omega}}(t)=$ 
$\left[\begin{array}{lll}\overline{\alpha^{(0)}}(t) & \ldots & \overline{\alpha^{(k)}}(t)\end{array}\right]^{\top}$. Using these notations, the following lemma is proposed:

Lemma 1: Let $\mathcal{A}(0) \in[\underline{\boldsymbol{\Omega}}(0), \overline{\boldsymbol{\Omega}}(0)]$. Let define $\forall 1 \leq j \leq k, \forall t$ :

$$
\begin{aligned}
& \frac{\alpha^{(j)}}{(t)}=\kappa_{j}(t)\left(\underline{\alpha^{(j-1)}}(t)-\alpha^{(j-1)}(t)\right)+\overparen{\alpha^{(j-1)}}(t) \\
& \overline{\alpha^{(j)}}(t)=\kappa_{j}(t)\left(\overline{\alpha^{(j-1)}}(t)-\alpha^{(j-1)}(t)\right)+\overparen{\overline{\alpha^{(j-1)}}}(t)
\end{aligned}
$$

Then,

$$
\alpha^{(k)}(t) \in\left[\underline{\alpha^{(k)}}(t), \overline{\alpha^{(k)}}(t)\right], \forall t \Rightarrow \alpha(t) \in[\underline{\alpha}(t), \bar{\alpha}(t)], \forall t
$$

Proof. See Appendix A.

Remark 3: As shown in Sect. 3.3, the OIST method consists in transforming output constraints into time-varying input saturations. It will be proved in Th. 1 that the introduction of the timevarying coefficients $\boldsymbol{\kappa}(t)$ in Eq. (21) is crucial to avoid the time-varying input constraints to overlap. Note that in the absence of unknown disturbances, these coefficients could be chosen positive constants, see Burlion (2012).

Remark 4: Considering Eq. (22), the notations are determining. For example, it should be clear that $\forall j, \forall t, \underline{\alpha}^{(j)}(t) \neq(\underline{\alpha})^{(j)}(t)$. More generally, and using notations defined later ${ }^{4}, \forall t, \underline{\boldsymbol{\Omega}}(t) \neq \underline{\mathcal{A}}(t)$. Moreover, while $\mathcal{A}(t)$ can be determined quite easily, this is much more difficult for $\underline{\boldsymbol{\Omega}}(t)$ since it depends on possibly unknown variables.

The main purpose of this lemma is to be able to satisfy the time-domain requirement on $\alpha(t)$ while setting constraints on $\alpha^{(k)}(t)$. Since the $k$-th derivative $\alpha^{(k)}(t)$ depends on $u$, this in turn leads to saturations on the control input signal which allow to fulfil $\alpha(t) \in[\underline{\alpha}(t), \bar{\alpha}(t)], \forall t$. This is presented in the next section.

Remark 5: Note this Lemma is still valid when introducing more conservative bounds $\underline{\beta^{j}}(t)$ and $\overline{\beta^{j}}(t)$ on $\alpha^{(j)}(t), \forall 1 \leq j \leq k$ :

$$
\forall t, \underline{\alpha^{(j)}}(t) \leq \underline{\beta^{j}}(t) \leq \alpha^{(j)}(t) \leq \overline{\beta^{j}}(t) \leq \overline{\alpha^{(j)}}(t)
$$

Of course, there is no more relation between these new bounds such as in Eq. (22). Their expressions can however be obtained using the expressions of the original bounds. This remark will be used in Sect. 3.5.1 to derive differentiable $\boldsymbol{\kappa}(t)$-coefficients from differentiable - yet more conservative bounds.

\subsection{Control saturations}

Given Assum. 3, there is $\mathbf{C}_{\alpha} \mathbf{A}^{k-1} \mathbf{B}_{u} \neq 0$. Let also suppose that $\mathbf{C}_{\alpha} \mathbf{A}^{k-1} \mathbf{B}_{u}>0$. Considering Lemma 1 and Eq. (20), and supposing that the expressions of $\alpha^{(k)}(t)$ and $\overline{\alpha^{(k)}}(t)$ are known $\forall t$, the saturations to apply to the control input $u$ are obtained in Eq. (24). In this case, it is also supposed that $d$ and $e$ are known signals:

\footnotetext{
${ }^{4}$ See Sect. 3.4 .
} 


$$
\begin{aligned}
& \underline{u}(t)=\frac{1}{\mathbf{C}_{\alpha} \mathbf{A}^{k-1} \mathbf{B}_{u}}\left[\frac{\alpha^{(k)}}{(t)}-\mathbf{C}_{\alpha} \mathbf{A}^{k} \boldsymbol{x}(t)-E_{\alpha} e^{(k)}(t)-\sum_{j=l}^{k} \mathbf{C}_{\alpha} \mathbf{A}^{j-1} \mathbf{B}_{d} d^{(k-j)}(t)\right] \\
& \bar{u}(t)=\frac{1}{\mathbf{C}_{\alpha} \mathbf{A}^{k-1} \mathbf{B}_{u}}\left[\overline{\alpha^{(k)}}(t)-\mathbf{C}_{\alpha} \mathbf{A}^{k} \boldsymbol{x}(t)-E_{\alpha} e^{(k)}(t)-\sum_{j=l}^{k} \mathbf{C}_{\alpha} \mathbf{A}^{j-1} \mathbf{B}_{d} d^{(k-j)}(t)\right]
\end{aligned}
$$

In case the disturbances $d$ and $e$ are unknown but Assum. 4 is satisfied, the following more conservative expressions are obtained:

$$
\begin{aligned}
& \underline{u}(t) \leq \underline{u}_{c}(t)=\frac{1}{\mathbf{C}_{\alpha} \mathbf{A}^{k-1} \mathbf{B}_{u}}\left[\underline{\alpha^{(k)}}(t)-\mathbf{C}_{\alpha} \mathbf{A}^{k} \boldsymbol{x}(t)+\left|E_{\alpha}\right| \max \left(\left|\underline{e^{(k)}}(t)\right|,\left|\overline{e^{(k)}}(t)\right|\right)\right. \\
& \left.+\sum_{j=l}^{k}\left|\mathbf{C}_{\alpha} \mathbf{A}^{j-1} \mathbf{B}_{d}\right| \max \left(\left|\underline{d^{(k-j)}}(t)\right|,\left|\overline{d^{(k-j)}}(t)\right|\right)\right] \\
& \bar{u}(t) \geq \bar{u}_{c}(t)=\frac{1}{\mathbf{C}_{\alpha} \mathbf{A}^{k-1} \mathbf{B}_{u}}\left[\overline{\alpha^{(k)}}(t)-\mathbf{C}_{\alpha} \mathbf{A}^{k} \boldsymbol{x}(t)-\left|E_{\alpha}\right| \max \left(\left|\underline{e^{(k)}}(t)\right|,\left|\overline{e^{(k)}}(t)\right|\right)\right. \\
& \left.-\sum_{j=l}^{k}\left|\mathbf{C}_{\alpha} \mathbf{A}^{j-1} \mathbf{B}_{d}\right| \max \left(\left|\underline{d^{(k-j)}}(t)\right|,\left|\overline{d^{(k-j)}}(t)\right|\right)\right]
\end{aligned}
$$

With a slight abuse of notation, the expressions from Eq. (25) will be used and denoted respectively $\underline{u}(t)$ and $\bar{u}(t)$. Before concluding this section, the following remark is made where it is observed that the expressions in Eq. (25) may be inconsistent due to the introduced conservatism:

Remark 6: For the saturations definition in Eq. (25) to be consistent and to avoid the control input saturations to overlap, one must ensure that $\underline{u}(t) \leq \bar{u}(t), \forall t$, i.e.:

$$
\overline{\alpha^{(k)}}(t)-\underline{\alpha^{(k)}}(t) \geq 2\left|E_{\alpha}\right| \max \left(\left|\underline{e^{(k)}}\right|,\left|\overline{e^{(k)}}\right|\right)+2 \sum_{j=l}^{k}\left|\mathbf{C}_{\alpha} \mathbf{A}^{j-1} \mathbf{B}_{d}\right| \max \left(\left|\underline{d^{(k-j)}}\right|,\left|\overline{d^{(k-j)}}\right|\right)
$$

Remark 7: In case $\mathbf{C}_{\alpha} \mathbf{A}^{k-1} \mathbf{B}_{u}<0$ and to avoid loss of generality, proper re-ordering of $\underline{u}(t)$ and $\bar{u}(t)$ is required. For a given input signal $u$, the saturating operator can be defined as follows:

$$
\operatorname{sat}(u)(t):=\max (\min (\underline{u}(t), \bar{u}(t)), \min (u(t), \max (\underline{u}(t), \bar{u}(t))))
$$

Proof. It is proved here that the operator $\operatorname{sat}(u)(t)$ as defined in Eq. (27) fulfils $\operatorname{sat}(u)(t) \in$ $[\underline{u}(t), \bar{u}(t)], \forall t:$

- suppose $\underline{u}(t)>\bar{u}(t), \forall t$. Then, $\min (\underline{u}(t), \bar{u}(t))=\bar{u}(t)$ and $\max (\underline{u}(t), \bar{u}(t))=\underline{u}(t)$. Thus, Eq. (27) becomes $\operatorname{sat}(u)(t)=\max (\bar{u}(t), \min (u(t), \underline{u}(t)))$;

- Suppose $\underline{u}(t)<\bar{u}(t), \forall t$. Then, $\min (\underline{u}(t), \bar{u}(t))=\underline{u}(t)$ and $\max (\underline{u}(t), \bar{u}(t))=\bar{u}(t)$. Thus, Eq. (27) becomes $\operatorname{sat}(u)(t)=\max (\underline{u}(t), \min (u(t), \bar{u}(t)))$.

In both cases, the expected result is obtained.

\subsection{Determination of bounds on the regulated variable derivatives}

In this section, an expression is established of the successive bounds on the derivatives of the regulated variable $\alpha$. This is done using the iterative definition given in Eq. (22) and supposing at 
first that $d$ and $e$ as well as their derivatives are known.

For any integer $j$ such that $1 \leq j \leq k$, let define the two following vectors:

- $\mathbf{U}^{j}(t)=\left[\begin{array}{lll}u_{0}^{j}(t) & \ldots & u_{k}^{j}(t)\end{array}\right] \in \mathbb{R}^{1 \times(k+1)}$ where $u_{j}^{j}(t)=1$ and $\forall i>j, u_{i}^{j}(t)=0$;

- $\mathbf{V}^{j}(t)=\left[\begin{array}{lll}v_{0}^{j}(t) & \ldots & v_{k-l-1}^{j}(t)\end{array}\right] \in \mathbb{R}^{1 \times(k-l)}$ where $\forall i>\max (-1, j-l-1), v_{i}^{j}(t)=0$ and

$$
v_{0}^{l+1}(t)=u_{l-1}^{l}(t) \mathbf{C}_{\alpha} \mathbf{A}^{l-1} \mathbf{B}_{d}
$$

Let $\underline{\mathcal{A}}=\left[\begin{array}{llll}\underline{\alpha} & \dot{\hat{\alpha}} & \ldots & (\underline{\alpha})^{(k)}\end{array}\right]^{\top} \in \mathbb{R}^{k+1}, \overline{\mathcal{A}}=\left[\begin{array}{llll}\bar{\alpha} & \dot{\bar{\alpha}} & \ldots & (\bar{\alpha})^{(k)}\end{array}\right]^{\top}$ and $\boldsymbol{\Theta}=$ $\left[\begin{array}{llll}\mathbf{C}_{\alpha} & \mathbf{C}_{\alpha} \mathbf{A} & \ldots & \mathbf{C}_{\alpha} \mathbf{A}^{k}\end{array}\right]^{\top} \in \mathbb{R}^{(k+1) \times n}$. Supposing $\mathcal{E}(t)$ and $\mathcal{D}(t)$ are known, one can express the bounds $\underline{\alpha^{(j)}}(t) \in \mathbb{R}$ and $\overline{\alpha^{(j)}}(t)$ to be satisfied by $\alpha^{(j)}(t), \forall t$, as

$$
\begin{aligned}
& \underline{\alpha^{(j)}}(t)=\boldsymbol{U}^{j}(t)\left\{\underline{\mathcal{A}}(t)-\boldsymbol{\Theta} x(t)-E_{\alpha} \mathcal{E}(t)\right\}+\mathbf{C}_{\alpha} \mathbf{A}^{j} \boldsymbol{x}(t)+E_{\alpha} e^{(j)}(t)-\boldsymbol{V}^{j}(t) \mathcal{D}(t) \\
& \overline{\alpha^{(j)}}(t)=\boldsymbol{U}^{j}(t)\left\{\overline{\mathcal{A}}(t)-\boldsymbol{\Theta} x(t)-E_{\alpha} \mathcal{E}(t)\right\}+\mathbf{C}_{\alpha} \mathbf{A}^{j} \boldsymbol{x}(t)+E_{\alpha} e^{(j)}(t)-\boldsymbol{V}^{j}(t) \mathcal{D}(t)
\end{aligned}
$$

Then, using Eq. (22), one can obtain the coefficients vectors $\boldsymbol{U}^{j}$ and $\boldsymbol{V}^{j}$ iteratively. This is illustrated in Eq. (29) where $\sigma\left(\boldsymbol{U}^{j-1}\right)(t)$ is the cyclic permutation of length $k+1$ on the elements of $\boldsymbol{U}^{j-1}(t)$, as defined in Def. 5 .

$$
\begin{aligned}
& \boldsymbol{U}^{0}(t)=\left[\begin{array}{llll}
1 & 0 & \ldots & 0
\end{array}\right] \\
& \forall j \text { s.t. } 1 \leq j \leq k, \quad \boldsymbol{U}^{j}(t)=\kappa_{j}(t) \boldsymbol{U}^{j-1}(t)+\dot{\boldsymbol{U}}^{j-1}(t)+\sigma\left(\boldsymbol{U}^{j-1}(t)\right) \\
& \forall j \text { s.t. } 0 \leq j \leq l, \quad V^{j}(t)=0 \\
& \forall j \text { s.t. } l<j \leq k, \quad \boldsymbol{V}^{j}(t)=\kappa_{j}(t)\left(\boldsymbol{V}^{j-1}(t)+\mathbf{C}_{\alpha} \mathbf{A}^{j-l-1}\left[\begin{array}{lll}
\mathbf{A}^{l-1} \mathbf{B}_{d} & \ldots & \mathbf{A}^{2 l-j} \mathbf{B}_{d}
\end{array}\right]\right)+\dot{\boldsymbol{V}}^{j-1}(t) \\
& +\sigma\left(\boldsymbol{V}^{j-1}(t)\right)+\left[\sum_{w=0}^{j-l-1} u_{l-1+w}^{j-1}(t) \mathbf{C}_{\alpha} \mathbf{A}^{l-1+w} \mathbf{B}_{d} \quad 0 \quad \ldots \quad 0\right]
\end{aligned}
$$

Proof. Tedious rewriting of Eq. (28) in explicit form and using Eq. (22) to express $\underline{\alpha^{(j+1)}}$ for $j \geq 0$ starting with $\underline{\alpha}^{(0)}(t)=\underline{\alpha}(t)$ leads to Eq. (29). The same calculus is performed as far as the upper bound is concerned.

Remark 8: Using the obtained iterative expressions and the fact that $u_{0}^{1}(t)=\kappa_{1}(t)$, one can determine that $\forall j, 0 \leq j \leq k, u_{j}^{j+1}(t)=\sum_{w=1}^{j+1} \kappa_{w}(t)$.

However, $\mathcal{E}(t)$ and $\mathcal{D}(t)$ are concatenations of unknown signals. By Assum. 4, only bounding signals are known on these disturbances. As stated in Remark 5, Lemma 1 is still valid if more conservative bounds are considered for application on the derivatives $\alpha^{(j)}(t)$. In case $\mathcal{E}(t)$ and $\mathcal{D}(t)$ are bounded by known bounds, the expressions in Eq. (28) become

$$
\begin{array}{r}
\underline{\beta^{j}}(t)=\boldsymbol{U}^{j}(t)\{\underline{\mathcal{A}}(t)-\boldsymbol{\Theta} \boldsymbol{x}(t)\}+\left|E_{\alpha}\right|\left|\boldsymbol{U}^{j}(t)\right| \max (|\underline{\mathcal{E}}(t)|,|\overline{\mathcal{E}}(t)|)+\mathbf{C}_{\alpha} \mathbf{A}^{j} \boldsymbol{x}(t) \\
\quad+\left|E_{\alpha}\right| \max \left(\left|\underline{e^{(j)}}(t)\right|,\left|\overline{e^{(j)}}(t)\right|\right)+\left|\boldsymbol{V}^{j}(t)\right| \max (|\underline{\mathcal{D}}(t)|,|\overline{\mathcal{D}}(t)|) \\
\overline{\beta^{j}}(t)=\boldsymbol{U}^{j}(t)\{\overline{\mathcal{A}}(t)-\boldsymbol{\Theta} \boldsymbol{x}(t)\}-\left|E_{\alpha}\right|\left|\boldsymbol{U}^{j}(t)\right| \max (|\underline{\mathcal{E}}(t)|,|\overline{\mathcal{E}}(t)|)+\mathbf{C}_{\alpha} \mathbf{A}^{j} \boldsymbol{x}(t) \\
-\left|E_{\alpha}\right| \max \left(\left|\underline{e^{(j)}}(t)\right|,\left|\overline{e^{(j)}}(t)\right|\right)-\left|\boldsymbol{V}^{j}(t)\right| \max (|\underline{\mathcal{D}}(t)|,|\overline{\mathcal{D}}(t)|)
\end{array}
$$


In case differentiable bounds are expected, the expressions in Eq. (31) are considered, where Def. 3 is used.

$$
\begin{aligned}
& \underline{\gamma^{j}}(t)=\boldsymbol{U}^{j}(t)\{\underline{\mathcal{A}}(t)-\boldsymbol{\Theta} \boldsymbol{x}(t)\}+\left|E_{\alpha}\right| f_{\mathrm{abs}}\left(\boldsymbol{U}^{j}(t)\right) g(\underline{\mathcal{E}}(t), \overline{\mathcal{E}}(t))+\mathbf{C}_{\alpha} \mathbf{A}^{j} \boldsymbol{x}(t) \\
& +\left|E_{\alpha}\right| g\left(\underline{e^{(j)}}(t), \overline{e^{(j)}}(t)\right)+f_{\text {abs }}\left(\boldsymbol{V}^{j}(t)\right) g(\underline{\mathcal{D}}(t), \overline{\mathcal{D}}(t)) \\
& \overline{\gamma^{j}}(t)=\boldsymbol{U}^{j}(t)\{\overline{\mathcal{A}}(t)-\boldsymbol{\Theta} \boldsymbol{x}(t)\}-\left|E_{\alpha}\right| f_{\text {abs }}\left(\boldsymbol{U}^{j}(t)\right) g(\underline{\mathcal{E}}(t), \overline{\mathcal{E}}(t))+\mathbf{C}_{\alpha} \mathbf{A}^{j} \boldsymbol{x}(t) \\
& -\left|E_{\alpha}\right| g\left(\underline{e^{(j)}}(t), \overline{e^{(j)}}(t)\right)-f_{\text {abs }}\left(\boldsymbol{V}^{j}(t)\right) g(\underline{\mathcal{D}}(t), \overline{\mathcal{D}}(t))
\end{aligned}
$$

where the following is fulfilled $\forall 0 \leq j \leq k, \forall t$ :

$$
\begin{aligned}
& \underline{\alpha^{(j)}}(t) \leq \underline{\beta^{j}}(t) \leq \underline{\gamma^{j}}(t) \\
& \overline{\alpha^{(j)}}(t) \geq \overline{\beta^{j}}(t) \geq \overline{\gamma^{j}}(t)
\end{aligned}
$$

which is compatible when used along with Lemma 1 as stated in Remark 5. However, the introduction of some conservatism requires to choose the coefficients $\boldsymbol{\kappa}(t)$ in $\boldsymbol{U}^{j}(t)$ and $\boldsymbol{V}^{j}(t)$ wisely. This is considered in the next section, along with other problems.

\subsection{Saturations analysis}

Using the expressions in Eq. (30) with $j=k$, the objective is to ensure $\alpha^{(k)}(t) \in\left[\underline{\beta^{k}}(t), \overline{\beta^{k}}(t)\right], \forall t$ so that, after using Lemma 1, the time-domain requirement is fulfilled:

$$
\begin{aligned}
& \underline{\beta^{k}}(t)=\boldsymbol{U}^{k}(t)\{\underline{\mathcal{A}}(t)-\boldsymbol{\Theta} \boldsymbol{x}(t)\}+\left|E_{\alpha}\right|\left|\boldsymbol{U}^{k}(t)\right| \max (|\underline{\mathcal{E}}(t)|,|\overline{\mathcal{E}}(t)|)+\mathbf{C}_{\alpha} \mathbf{A}^{k} \boldsymbol{x}(t) \\
& +\left|E_{\alpha}\right| \max \left(\left|\underline{e}^{(k)}(t)\right|,\left|\overline{e^{(k)}}(t)\right|\right)+\left|\boldsymbol{V}^{k}(t)\right| \max (|\underline{\mathcal{D}}(t)|,|\overline{\mathcal{D}}(t)|) \\
& \overline{\beta^{k}}(t)=\boldsymbol{U}^{k}(t)\{\overline{\mathcal{A}}(t)-\boldsymbol{\Theta} \boldsymbol{x}(t)\}-\left|E_{\alpha}\right|\left|\boldsymbol{U}^{k}(t)\right| \max (|\underline{\mathcal{E}}(t)|,|\overline{\mathcal{E}}(t)|)+\mathbf{C}_{\alpha} \mathbf{A}^{k} \boldsymbol{x}(t) \\
& -\left|E_{\alpha}\right| \max \left(\left|\underline{e^{(k)}}(t)\right|,\left|\overline{e^{(k)}}(t)\right|\right)-\left|\boldsymbol{V}^{k}(t)\right| \max (|\underline{\mathcal{D}}(t)|,|\overline{\mathcal{D}}(t)|)
\end{aligned}
$$

These expressions are then used in Eq. (25) in place of $\underline{\alpha^{(k)}}(t)$ and $\overline{\alpha^{(k)}}(t)$ to obtain explicit expressions of the control input saturations:

$$
\begin{aligned}
\underline{u}(t)=\frac{1}{\mathbf{C}_{\alpha} \mathbf{A}^{k-1} \mathbf{B}_{u}}\left[\boldsymbol{U}^{k}(t)\{\underline{\mathcal{A}}(t)-\boldsymbol{\Theta} \boldsymbol{x}(t)\}+\left|E_{\alpha}\right|\left|\boldsymbol{U}^{k}(t)\right| \max (|\underline{\mathcal{E}}(t)|,|\overline{\mathcal{E}}(t)|)\right. \\
+2\left|E_{\alpha}\right| \max \left(\left|\underline{e^{(k)}}(t)\right|,\left|\overline{e^{(k)}}(t)\right|\right)+\left|\boldsymbol{V}^{k}(t)\right| \max (|\underline{\mathcal{D}}(t)|,|\overline{\mathcal{D}}(t)|) \\
\left.+\sum_{j=l}^{k}\left|\mathbf{C}_{\alpha} \mathbf{A}^{j-1} \mathbf{B}_{d}\right| \max \left(\left|\underline{d^{(k-j)}}(t)\right|,\left|\overline{d^{(k-j)}}(t)\right|\right)\right] \\
\bar{u}(t)=\frac{1}{\mathbf{C}_{\alpha} \mathbf{A}^{k-1} \mathbf{B}_{u}}\left[\boldsymbol{U}^{k}(t)\right. \\
\left.-2\left|E_{\alpha}\right| \max (t)-\boldsymbol{\Theta} \boldsymbol{x}(t)\right\}-\left|E_{\alpha}\right|\left|\boldsymbol{U}^{k}(t)\right| \max (|\underline{\mathcal{E}}(t)|,|\overline{\mathcal{E}}(t)|) \\
\left.-\sum_{j=l}^{k}\left|\mathbf{C}_{\alpha} \mathbf{A}^{j-1} \mathbf{B}_{d}\right| \max \left(\left|\underline{d^{(k-j)}}(t)\right|,\left|\overline{d^{(k-j)}}(t)\right|\right)\right] \\
\end{aligned}
$$


These now depend on the design parameters $\boldsymbol{\kappa}(t)$ from Eq. (21), $\mathcal{A}(t)$ and $\overline{\mathcal{A}}(t)$ and on the signals describing the limited knowledge of the disturbances: $\underline{\mathcal{D}}(t), \overline{\mathcal{D}}(t), \underline{\mathcal{E}}(t)$ and $\overline{\mathcal{E}}(t)$. As suggested by Remark 6 , some further analysis is however required. It is particularly important to detect possible saturations overlap - by choosing the $\boldsymbol{\kappa}(t)$-signals wisely - or to study the reachability of the origin - a result which will be used to prove the closed-loop stability.

\subsubsection{Saturations overlap}

Let define $\forall t, \Delta_{0}(t):=\bar{\alpha}(t)-\underline{\alpha}(t)$ and, $\forall 1 \leq j \leq k, \Delta_{j}(t):=\overline{\beta^{j}}(t)-\underline{\beta^{j}}(t)$. By Assumption 1, $\Delta_{0}(t)>0$ is satisfied $\forall t$. Thus,

$$
\begin{aligned}
\Delta_{j}(t)=\boldsymbol{U}^{j}(t)\{\overline{\mathcal{A}}(t)-\underline{\mathcal{A}}(t)\}-2\left|E_{\alpha}\right|\left|\boldsymbol{U}^{j}(t)\right| \max (|\mathcal{\mathcal { E }}(t)|,|\overline{\mathcal{E}}(t)|) \\
\quad-2\left|\boldsymbol{V}^{j}(t)\right| \max (|\underline{\mathcal{D}}(t)|,|\overline{\mathcal{D}}(t)|)-2\left|E_{\alpha}\right| \max \left(\left|\underline{e^{(j)}}(t)\right|,\left|\overline{e^{(j)}}(t)\right|\right)
\end{aligned}
$$

Using Eq. (30) and the fact that the signals in $\boldsymbol{\kappa}(t)$ are chosen positive by construction (see below), the dependence on $\kappa_{j}(t)$ can be made explicit, $\forall 1 \leq j \leq k, \forall t$ :

$$
\begin{aligned}
& \Delta_{j}(t) \geq \tilde{\Delta}_{j}(t)=\kappa_{j}(t)\left[\boldsymbol{U}^{j-1}(t)\{\overline{\mathcal{A}}(t)-\underline{\mathcal{A}}(t)\}-2\left|E_{\alpha}\right|\left|\boldsymbol{U}^{j-1}(t)\right| \max (|\underline{\mathcal{E}}(t)|,|\overline{\mathcal{E}}(t)|)\right. \\
& \left.-2\left|\boldsymbol{V}^{j-1}(t)+\mathbf{C}_{\alpha} \mathbf{A}^{j-l-1}\left[\begin{array}{lll}
\mathbf{A}^{l-1} \mathbf{B}_{d} & \ldots & \mathbf{A}^{2 l-k} \mathbf{B}_{d}
\end{array}\right]\right| \max (|\underline{\mathcal{D}}(t)|,|\overline{\mathcal{D}}(t)|)\right] \\
& +\left[\dot{\boldsymbol{U}}^{j-1}(t)+\sigma\left(\boldsymbol{U}^{j-1}(t)\right)\right]\{\overline{\mathcal{A}}(t)-\underline{\mathcal{A}}(t)\}-2\left|E_{\alpha}\right|\left|\dot{\boldsymbol{U}}^{j-1}(t)+\sigma\left(\boldsymbol{U}^{j-1}(t)\right)\right| \max (|\underline{\mathcal{E}}(t)|,|\overline{\mathcal{E}}(t)|) \\
& -2\left|E_{\alpha}\right| \max \left(\left|\frac{e^{(j)}}{}(t)\right|,\left|\overline{e^{(j)}}(t)\right|\right)-2 \mid \dot{\boldsymbol{V}}^{j-1}(t)+\sigma\left(\boldsymbol{V}^{j-1}(t)\right)+ \\
& {\left[\sum_{w=0}^{j-l-1} u_{l-1+w}^{j-1}(t) \mathbf{C}_{\alpha} \mathbf{A}^{l-1+w} \mathbf{B}_{d} \quad 0 \quad \ldots \quad 0\right] \mid \max (|\underline{\mathcal{D}}(t)|,|\overline{\mathcal{D}}(t)|)}
\end{aligned}
$$

which is, in turn, less conservative than the differentiable approximate:

$$
\begin{aligned}
& \tilde{\Delta}_{j}(t) \geq \widehat{\Delta}_{j}(t)=\kappa_{j}(t)\left[\boldsymbol{U}^{j-1}(t)\{\overline{\mathcal{A}}(t)-\underline{\mathcal{A}}(t)\}-2\left|E_{\alpha}\right| f_{\text {abs }}\left(\boldsymbol{U}^{j-1}(t)\right) g(\underline{\mathcal{E}}(t), \overline{\mathcal{E}}(t))\right. \\
& \left.-2 f_{\text {abs }}\left(\boldsymbol{V}^{j-1}(t)+\mathbf{C}_{\alpha} \mathbf{A}^{j-l-1}\left[\begin{array}{lll}
\mathbf{A}^{l-1} \mathbf{B}_{d} & \ldots & \mathbf{A}^{2 l-k} \mathbf{B}_{d}
\end{array}\right]\right) g(\underline{\mathcal{D}}(t), \overline{\mathcal{D}}(t))\right] \\
& +\left[\dot{\boldsymbol{U}}^{j-1}(t)+\sigma\left(\boldsymbol{U}^{j-1}(t)\right)\right]\{\overline{\mathcal{A}}(t)-\underline{\mathcal{A}}(t)\}-2\left|E_{\alpha}\right| f_{\text {abs }}\left(\dot{\boldsymbol{U}}^{j-1}(t)+\sigma\left(\boldsymbol{U}^{j-1}(t)\right)\right) g(\underline{\mathcal{E}}(t), \overline{\mathcal{E}}(t)) \\
& -2\left|E_{\alpha}\right| g\left(\underline{e^{(j)}}(t), \overline{e^{(j)}}(t)\right)-2 f_{\text {abs }}\left(\dot{\boldsymbol{V}}^{j-1}(t)+\sigma\left(\boldsymbol{V}^{j-1}(t)\right)+\right. \\
& \left.\left[\begin{array}{lllll}
\sum_{w=0}^{j-l-1} u_{l-1+w}^{j-1}(t) \mathbf{C}_{\alpha} \mathbf{A}^{l-1+w} \mathbf{B}_{d} & 0 & \ldots & 0
\end{array}\right]\right) g(\underline{\mathcal{D}}(t), \overline{\mathcal{D}}(t))
\end{aligned}
$$

which is simplified into

$$
\widehat{\Delta}_{j}(t)=\kappa_{j}(t) \widehat{\lambda}_{j}^{d}(t)+\widehat{\lambda}_{j}^{n}(t)
$$

where $\widehat{\lambda}_{j}^{d}(t)$ and $\widehat{\lambda}_{j}^{d}(t)$ only depend on $\kappa_{l}(t)$ with $l<j$. Considering Remark 6 , the following lemma is proposed:

Lemma 2: Saturations overlap is avoided if 
- $\Delta_{0}(t) \geq 0, \forall t$, which is true by Assumption 1;

- $\forall 1 \leq j<k, \widehat{\Delta}_{j}(t) \geq 0, \forall t$;

- $\widehat{\Delta}_{k}(t) \geq 2\left|E_{\alpha}\right| \max \left(\left|\underline{e^{(k)}}\right|,\left|\overline{e^{(k)}}\right|\right)+2 \sum_{j=l}^{k}\left|\mathbf{C}_{\alpha} \mathbf{A}^{j-1} \mathbf{B}_{d}\right| \max \left(\left|\underline{d^{(k-j)}}\right|,\left|\overline{d^{(k-j)}}\right|\right), \forall t$.

The last condition ensures that $\bar{u}(t)-\underline{u}(t) \geq 0, \forall t$. A constructive definition of the coefficients $\kappa_{j}(t)$ in Eq. (21) can be used to fulfil Lemma 2.

Theorem 1: Saturations overlap is avoided if

- Assumption 1 is satisfied, i.e. $\bar{\alpha}(t) \geq \underline{\alpha}(t)$ and $\widehat{\lambda}_{1}^{d}(t) \neq 0, \forall t$;

- $\forall 1 \leq j<k$, one ensures $\widehat{\Delta}_{j}(t) \geq 0$ by choosing, $\forall t$,

$$
\kappa_{j}(t)=\frac{\breve{\kappa}_{j}-\widehat{\lambda}_{j}^{n}(t)}{\widehat{\lambda}_{j}^{d}(t)}
$$

where $\breve{\kappa}_{j}>0$ is chosen such that $\kappa_{1}(t)>\frac{1}{2}, \forall j>1, \kappa_{j}(t)>1$ and $\widehat{\lambda}_{j+1}^{d}(t) \neq 0, \forall t$.

- for $j=k$, one ensures

$$
\widehat{\Delta}_{k}(t) \geq 2\left|E_{\alpha}\right| \max \left(\left|\underline{e^{(k)}}\right|,\left|\overline{e^{(k)}}\right|\right)+2 \sum_{j=l}^{k}\left|\mathbf{C}_{\alpha} \mathbf{A}^{j-1} \mathbf{B}_{d}\right| \max \left(\left|\underline{d^{(k-j)}}\right|,\left|\overline{d^{(k-j)}}\right|\right)
$$

by choosing, $\forall t$,

$$
\kappa_{k}(t)=\frac{\breve{\kappa}_{k}-\widehat{\lambda}_{k}^{n}(t)+2\left|E_{\alpha}\right| \max \left(\left|\underline{e}^{(k)}\right|,\left|\overline{e^{(k)}}\right|\right)+2 \sum_{j=l}^{k}\left|\mathbf{C}_{\alpha} \mathbf{A}^{j-1} \mathbf{B}_{d}\right| \max \left(\left|\underline{d^{(k-j)}}\right|,\left|\overline{d^{(k-j)}}\right|\right)}{\widehat{\lambda}_{k}^{d}(t)}
$$

where $\breve{\kappa}_{k}>0$ is chosen such that $\kappa_{k}(t)>\frac{1}{2}, \forall t$.

Proof. Straightforward using Lemma 2 and Eq. (38). As far as the minimal values for the $\kappa_{j}(t)$ are concerned, this is discussed in the proof of Prop. 2, in Appendix B.

Remark 9: Note that $\kappa_{k}(t)$ is not differentiable with respect to $t$ by definition but this is not required contrary to the other coefficients.

By the end of this section, a vector of design parameters $\breve{\boldsymbol{\kappa}}=\left[\begin{array}{lll}\breve{\kappa}_{1} & \ldots & \breve{\kappa}_{k}\end{array}\right]$ is obtained, ensuring no overlap and the differentiability of $\boldsymbol{\kappa}(t)$ (to the exception of $\kappa_{k}(t)$ ).

\subsubsection{State-independent saturations}

It is interesting to note that only one term depends on the state vector $x(t)$ in the expressions of the control input saturations obtained in Eq. (34). Let define:

$$
\forall t, \boldsymbol{K}_{\text {oist }}(t):=\frac{\boldsymbol{U}^{k}(t) \Theta}{\mathbf{C}_{\alpha} \mathbf{A}^{k-1} \mathbf{B}_{u}} \in \mathbb{R}^{1 \times n}
$$

It is observed that introducing saturations on the control input $u$ is equivalent to saturating the signal $v$ defined as: 


$$
v(t):=u(t)+\boldsymbol{K}_{\text {oist }}(t) \boldsymbol{y}(t)=u(t)+\boldsymbol{K}_{\text {oist }}(t) \boldsymbol{x}(t)+\boldsymbol{K}_{\text {oist }}(t) \mathbf{D}_{e} e(t), \forall t
$$

by the following state-free saturations:

$$
\begin{aligned}
& \underline{v}(t)=\underline{u}(t)+\boldsymbol{K}_{\text {oist }}(t) \boldsymbol{x}(t)+\left|\boldsymbol{K}_{\text {oist }}(t) \mathbf{D}_{e}\right| \max (|\underline{e}(t)|,|\bar{e}(t)|) \\
& \bar{v}(t)=\bar{u}(t)+\boldsymbol{K}_{\text {oist }}(t) \boldsymbol{x}(t)-\left|\boldsymbol{K}_{\text {oist }}(t) \mathbf{D}_{e}\right| \max (|\underline{e}(t)|,|\bar{e}(t)|)
\end{aligned}
$$

or, more precisely

$$
\begin{aligned}
& \underline{v}(t)=\frac{1}{\mathbf{C}_{\alpha} \mathbf{A}^{k-1} \mathbf{B}_{u}}\left[\boldsymbol{U}^{k}(t) \underline{\mathcal{A}}(t)+\left|E_{\alpha}\right|\left|\boldsymbol{U}^{k}(t)\right| \max (|\underline{\mathcal{E}}(t)|,|\overline{\mathcal{E}}(t)|)+2\left|E_{\alpha}\right| \max \left(\left|\underline{e}^{(k)}(t)\right|,\left|\overline{e^{(k)}}(t)\right|\right)\right. \\
& \left.+\left|\boldsymbol{V}^{k}(t)\right| \max (|\underline{\mathcal{D}}(t)|,|\overline{\mathcal{D}}(t)|)+\sum_{j=l}^{k}\left|\mathbf{C}_{\alpha} \mathbf{A}^{j-1} \mathbf{B}_{d}\right| \max \left(\left|\underline{d^{(k-j)}}(t)\right|,\left|\overline{d^{(k-j)}}(t)\right|\right)\right] \\
& +\left|\boldsymbol{K}_{\text {oist }}(t) \mathbf{D}_{e}\right| \max (|\underline{e}(t)|,|\bar{e}(t)|) \\
& \bar{v}(t)=\frac{1}{\mathbf{C}_{\alpha} \mathbf{A}^{k-1} \mathbf{B}_{u}}\left[\boldsymbol{U}^{k}(t) \overline{\mathcal{A}}(t)-\left|E_{\alpha}\right|\left|\boldsymbol{U}^{k}(t)\right| \max (|\underline{\mathcal{E}}(t)|,|\overline{\mathcal{E}}(t)|)-2\left|E_{\alpha}\right| \max \left(\left|\underline{e^{(k)}}(t)\right|,\left|\overline{e^{(k)}}(t)\right|\right)\right. \\
& \left.-\left|\boldsymbol{V}^{k}(t)\right| \max (|\underline{\mathcal{D}}(t)|,|\overline{\mathcal{D}}(t)|)-\sum_{j=l}^{k}\left|\mathbf{C}_{\alpha} \mathbf{A}^{j-1} \mathbf{B}_{d}\right| \max \left(\left|\underline{d^{(k-j)}}(t)\right|,\left|\overline{d^{(k-j)}}(t)\right|\right)\right] \\
& -\left|\boldsymbol{K}_{\text {oist }}(t) \mathbf{D}_{e}\right| \max (|\underline{e}(t)|,|\bar{e}(t)|)
\end{aligned}
$$

Remark 10: One now has to ensure that $\bar{v}(t)-\underline{v}(t) \geq 0, \forall t$ using similar considerations than in Th. 1.

Using $v$ instead of $u$ as the new input to system $(G)$ in Eq. (12), the saturated system becomes

$$
\dot{\boldsymbol{x}}(t)=\left[\mathbf{A}-\mathbf{B}_{u} \boldsymbol{K}_{\text {oist }}(t)\right] \boldsymbol{x}(t)+\mathbf{B}_{u} \mathrm{sat}_{\underline{v}(t)}^{\bar{v}(t)}(v(t))-\mathbf{B}_{u} \boldsymbol{K}_{\text {oist }}(t) \mathbf{D}_{e} e(t)+\mathbf{B}_{d} d(t)
$$

\subsubsection{Admissible asymptotic equilibrium}

Using Eq. (34) and the definitions of the state-free saturations in Eq. (43) the saturations in Eq. (44) were obtained. Using Assum. 2, it comes that:

$$
\lim _{t \rightarrow \infty} \mathcal{A}(t)=\left[\begin{array}{llll}
\underline{\alpha}^{\star} & 0 & \ldots & 0
\end{array}\right]^{\top}, \lim _{t \rightarrow \infty} \overline{\mathcal{A}}(t)=\left[\begin{array}{llll}
\bar{\alpha}^{\star} & 0 & \ldots & 0
\end{array}\right]
$$

Considering Th. 1 along with Assum. 5, it can be observed that the design signal $\boldsymbol{\kappa}(t)$ in Eq. (21) converges towards a constant value. Thus, this is also the case of vectors $\boldsymbol{U}^{j}(t)$ and $\boldsymbol{V}^{j}(t), \forall 1 \leq j \leq$ $k$, and $\lim _{t \rightarrow \infty} \boldsymbol{K}_{\text {oist }}(t)=\boldsymbol{K}_{\text {oist }}^{\star}$. Consequently, as far as the saturations in Eq. (44) are concerned and using Assum. 4, they tend towards the following limits: 


$$
\begin{array}{r}
\underline{v}^{\star}=\frac{1}{\mathbf{C}_{\alpha} \mathbf{A}^{k-1} \mathbf{B}_{u}}\left[u_{0}^{k, \star} \underline{\alpha}^{\star}+\left|E_{\alpha}\right|\left|u_{0}^{k, \star}\right| \max \left(\left|\underline{e}^{\star}\right|,\left|\bar{e}^{\star}\right|\right)+\left\{\left|v_{0}^{k, \star}\right|+\left|\mathbf{C}_{\alpha} \mathbf{A}^{k-1} \mathbf{B}_{d}\right|\right\} \max \left(\left|\underline{d}^{\star}\right|,\left|\bar{d}^{\star}\right|\right)\right] \\
+\left|\boldsymbol{K}_{\text {oist }}^{\star} \mathbf{D}_{e}\right| \max \left(\left|\underline{e}^{\star}\right|,\left|\bar{e}^{\star}\right|\right) \\
\bar{v}^{\star}=\frac{1}{\mathbf{C}_{\alpha} \mathbf{A}^{k-1} \mathbf{B}_{u}}\left[u_{0}^{k, \star} \bar{\alpha}^{\star}-\left|E_{\alpha}\right|\left|u_{0}^{k, \star}\right| \max \left(\left|\underline{e}^{\star}\right|,\left|\bar{e}^{\star}\right|\right)-\left\{\left|v_{0}^{k, \star}\right|+\left|\mathbf{C}_{\alpha} \mathbf{A}^{k-1} \mathbf{B}_{d}\right|\right\} \max \left(\left|\underline{d}^{\star}\right|,\left|\bar{d}^{\star}\right|\right)\right] \\
-\left|\boldsymbol{K}_{\text {oist }}^{\star} \mathbf{D}_{e}\right| \max \left(\left|\underline{e}^{\star}\right|,\left|\bar{e}^{\star}\right|\right)
\end{array}
$$

and the unsaturated state-free control becomes ${ }^{5}$ :

$$
v^{\star}=\mathbf{C}_{K} \boldsymbol{x}_{K}^{\star}+\left(\mathbf{D}_{K}+\boldsymbol{K}_{\text {oist }}^{\star}\right)\left(\boldsymbol{x}^{\star}+\mathbf{D}_{\mathbf{e}} e^{\star}\right)=0
$$

In the following proposition it is shown that the origin is an admissible equilibrium under some condition. This condition can be evaluated during the analysis phase of the unconstrained closedloop system.

Proposition 1: Let $\boldsymbol{x}^{\star} \in \mathbb{R}^{n}$ and suppose Assum. 2, 4 and 5 are satisfied. In the non-restrictive case where $\boldsymbol{x}^{\star}=0$, this is an admissible asymptotic equilibrium if

$$
v^{\star}=0 \in\left[\underline{v}^{\star}, \bar{v}^{\star}\right]
$$

or, more precisely, if:

$$
\begin{gathered}
\frac{1}{\mathbf{C}_{\alpha} \mathbf{A}^{k-1} \mathbf{B}_{u}}\left[u_{0}^{k, \star} \underline{\alpha}^{\star}+\left|E_{\alpha}\right|\left|u_{0}^{k, \star}\right| \max \left(\left|\underline{e}^{\star}\right|,\left|\bar{e}^{\star}\right|\right)+\left\{\left|v_{0}^{k, \star}\right|+\left|\mathbf{C}_{\alpha} \mathbf{A}^{k-1} \mathbf{B}_{d}\right|\right\} \max \left(\left|\underline{d}^{\star}\right|,\left|\bar{d}^{\star}\right|\right)\right] \\
+\left|\boldsymbol{K}_{\text {oist }}^{\star} \mathbf{D}_{e}\right| \max \left(\left|\underline{e}^{\star}\right|,\left|\bar{e}^{\star}\right|\right) \leq 0 \leq-\left|\boldsymbol{K}_{\text {oist }}^{\star} \mathbf{D}_{e}\right| \max \left(\left|\underline{e}^{\star}\right|,\left|\bar{e}^{\star}\right|\right) \\
+\frac{1}{\mathbf{C}_{\alpha} \mathbf{A}^{k-1} \mathbf{B}_{u}}\left[u_{0}^{k, \star} \bar{\alpha}^{\star}-\left|E_{\alpha}\right|\left|u_{0}^{k, \star}\right| \max \left(\left|\underline{e}^{\star}\right|,\left|\bar{e}^{\star}\right|\right)-\left\{\left|v_{0}^{k, \star}\right|+\left|\mathbf{C}_{\alpha} \mathbf{A}^{k-1} \mathbf{B}_{d}\right|\right\} \max \left(\left|\underline{d}^{\star}\right|,\left|\bar{d}^{\star}\right|\right)\right]
\end{gathered}
$$

Proof. Straightforward using the asymptotic results in Eq. (47) and (48).

Using this analysis on the saturations, the stability of the system in closed-loop with the saturated nominal controller is studied in the next section.

\section{Closed-loop stability}

In this section, the stability of the system is proved in closed-loop with the saturated control signal $\operatorname{sat}_{\underline{u}(t)}^{\bar{u}(t)}\left(\mathbf{C}_{K} \boldsymbol{x}_{K}(t)+\mathbf{D}_{K} \boldsymbol{y}(t)\right)$, where $\underline{u}(t)$ and $\bar{u}(t)$ have been obtained using the approach presented in Sect. 3 .

\footnotetext{
${ }^{5}$ In the non-pathological case where $e$ is a physical signal converging to zero under Assum. 5.
} 


\subsection{Considered system}

As mentioned in Sect. 3.5.2, the control signal saturations depend on the system state $x(t)$. Changing the control signal into $v(t)=u(t)+\boldsymbol{K}_{\text {oist }}(t) \boldsymbol{y}(t)$, the system studied in this section is equivalent to the system in Eq. (51) where the saturations on $v(t)$ do not depend on the state vector anymore.

$$
(G)\left\{\begin{array}{l}
\dot{\boldsymbol{x}}(t)=\left[\mathbf{A}-\mathbf{B}_{u} \boldsymbol{K}_{\mathrm{oist}}(t)\right] \boldsymbol{x}(t)+\mathbf{B}_{u} \mathrm{sat}_{\underline{v}(t)}^{\bar{v}(t)}(v(t))-\mathbf{B}_{u} \boldsymbol{K}_{\mathrm{oist}}(t) \mathbf{D}_{e} e(t)+\mathbf{B}_{d} d(t) \\
\boldsymbol{y}(t)=\boldsymbol{x}(t)+\mathbf{D}_{e} e(t) \\
\dot{\boldsymbol{x}}_{K}(t)=\mathbf{A}_{K} \boldsymbol{x}_{K}(t)+\mathbf{B}_{K} \boldsymbol{y}(t) \\
v(t)=\mathbf{C}_{K} \boldsymbol{x}_{K}(t)+\left(\mathbf{D}_{K}+\boldsymbol{K}_{\text {oist }}(t)\right) \boldsymbol{y}(t) \\
\alpha(t)=\mathbf{C}_{\alpha} \boldsymbol{x}(t)+E_{\alpha} e(t)
\end{array}\right.
$$

The following lemma will be used to demonstrate the final theorem of this paper:

Lemma 3: $\forall \boldsymbol{x}(t) \in \mathbb{R}^{n}, \forall t$, the function $-\boldsymbol{K}_{\text {oist }}(t) \boldsymbol{x}(t)$ is Lipschitz (with respect to $\boldsymbol{x}$ ). Moreover, $-\boldsymbol{K}_{\text {oist }}(t) \boldsymbol{x}(t)$ is $K_{1}$-Lipschitz, where $K_{1}=\max _{t}\left\|\boldsymbol{K}_{\text {oist }}(t)\right\| \in \mathbb{R}$.

Proof. Let define, $\forall \boldsymbol{x} \in \mathbb{R}^{n}, \forall t, f(\boldsymbol{x}, t)=-\boldsymbol{K}_{\text {oist }}(t) \boldsymbol{x}(t)$. Then, $\forall \boldsymbol{x} \in \mathbb{R}^{n}, \frac{\partial f}{\partial \boldsymbol{x}}(\boldsymbol{x}, t)=$ $-\boldsymbol{K}_{\text {oist }}(t)$. Since $\boldsymbol{K}_{\text {oist }}(t)$ is continuous $\forall t$ by definition of the coefficients $\boldsymbol{\kappa}(t)$ and continuity of $[\underline{\mathcal{D}}(t), \overline{\mathcal{D}}(t), \underline{\mathcal{E}}(t), \overline{\mathcal{E}}(t)]$ (see Assum. 4), the function $f$ is continuously differentiable with respect to the state $\boldsymbol{x}$. This implies that $\boldsymbol{K}_{\text {oist }}(t) \boldsymbol{x}(t)$ is a Lipschitz continuous function with respect to $\boldsymbol{x}$.

\section{$4.2 \quad$ Closed-loop stability using an anti-windup approach}

Due to the presence of a dynamic controller and saturations, unexpected closed-loop behaviour is expected. Anti-windup techniques have been widely studied and used to avoid behaviours like controller state divergence. Some of these techniques are presented in Grimm et al. (2003); Kapoor, Teel, and Daoutidis (1998); Tarbouriech and Turner (2009). The approach proposed in Menon, Herrmann, Turner, Bates, and Postlethwaite (2006) and Herrmann, Menon, Turner, Bates, and Postlethwaite (2010) deals with a specific class of nonlinear systems to which the system presented in Eq. (51) belongs. In this article, the anti-windup framework is used to prove the closed-loop stability of the system in Eq. (51) where the time-varying gain $\boldsymbol{K}_{\text {oist }}(t)$ and saturations are respectively given by Eq. (41) and Eq. (44).

Proposition 2: The open-loop system

$$
\dot{\boldsymbol{x}}(t)=\left[\mathbf{A}-\mathbf{B}_{u} \boldsymbol{K}_{\text {oist }}(t)\right] \boldsymbol{x}(t)
$$

is GES.

The proof is inspired by Herrmann et al. (2010).

Proof. See Appendix B.

To ensure asymptotic stability of the closed-loop containing the dynamic controller, it is necessary to introduce an anti-windup. Considering the system in Eq. (51), the following anti-windup with state $x_{a} \in \mathbb{R}^{n}$ is introduced 


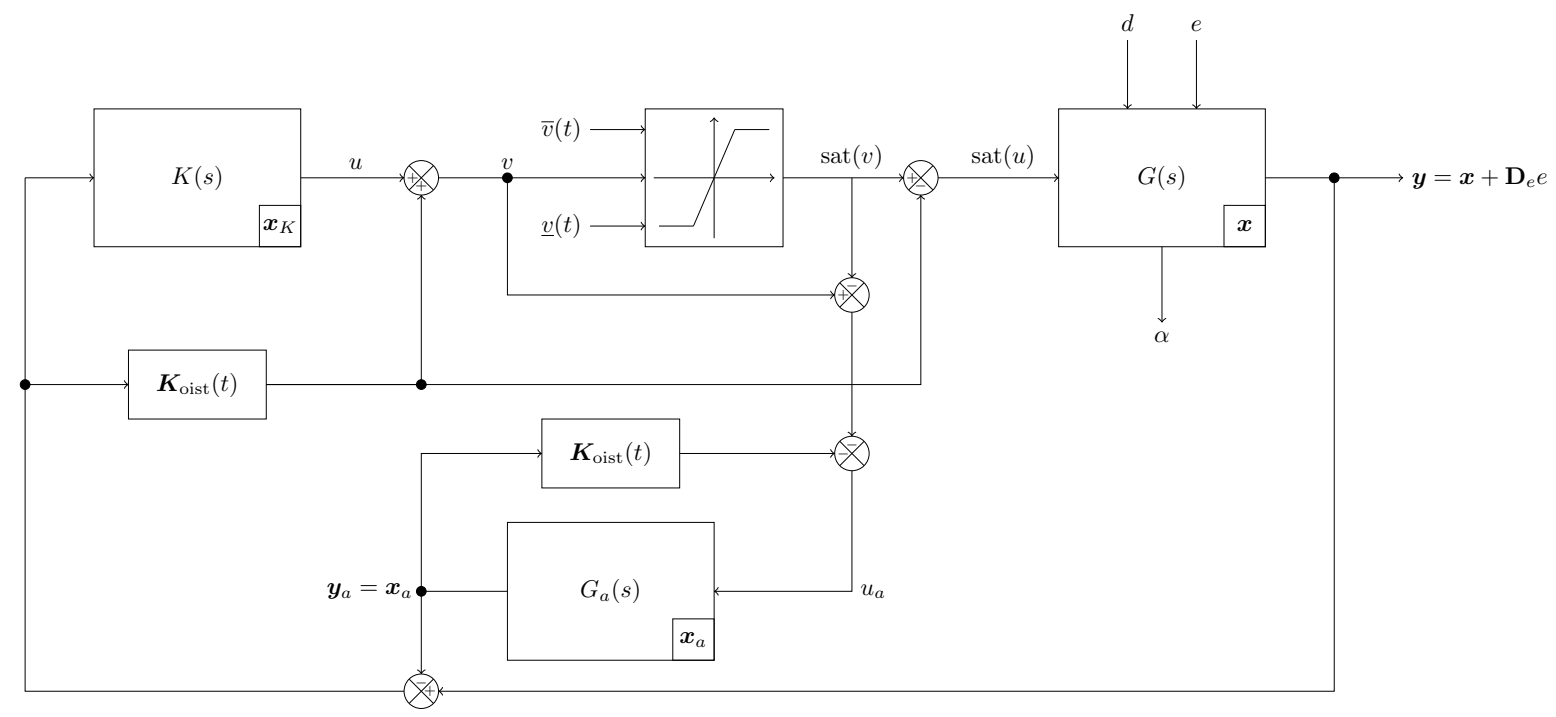

Figure 8. Closed-loop illustration.

$$
\left(G_{a}\right)\left\{\begin{array}{l}
\dot{\boldsymbol{x}}_{a}(t)=\mathbf{A} \boldsymbol{x}_{a}(t)+\mathbf{B}_{u} u_{a}(t) \\
\boldsymbol{y}_{a}=\boldsymbol{x}_{a} \\
u_{a}(t)=-\boldsymbol{K}_{\mathrm{oist}}(t) \boldsymbol{y}_{a}(t)-\mathrm{Dz}_{\underline{v}} \bar{v}(t) \\
v_{1}(t)=-\left[K(s)+\boldsymbol{K}_{\mathrm{oist}}(t)\right] \boldsymbol{y}_{a}(t)
\end{array}\right.
$$

The control $v$ is then modified into

$$
\begin{aligned}
v(t) & =u(t)+\boldsymbol{K}_{\mathrm{oist}}(t) \boldsymbol{y}(t)+v_{1}(t) \\
& =\mathbf{C}_{K} \boldsymbol{x}_{K}(t)+\mathbf{D}_{K}\left(\boldsymbol{y}(t)-\boldsymbol{y}_{a}(t)\right)+\boldsymbol{K}_{\mathrm{oist}}(t)\left(\boldsymbol{y}(t)-\boldsymbol{y}_{a}(t)\right)
\end{aligned}
$$

The main result of this section is the following theorem which proves stability of the system in closed-loop with the saturated nominal controller. Both this theorem and its proof are inspired from Menon et al. (2006) and Herrmann et al. (2010).

Theorem 2: If Assum. 1 to 8 are satisfied ${ }^{6}$ (resulting in Th. 1 and Prop. 2), the origin of the closed-loop system consisting of the system in Eq. (45) - where the time-varying saturations are given in Eq. (44) - the control law in Eq. (54) and the anti-windup compensator given in Eq. (53) is $G A S$.

Proof. See Appendix C.

An illustration of the system in closed-loop with the anti-windup and saturating block is given in Fig. 8. This is the typical structure obtained when implementing OIST on a linear system.

${ }^{6}$ And in the non-pathological case where $d$ is a non-converging finite energy distribution, which is not a realistic physical case. 

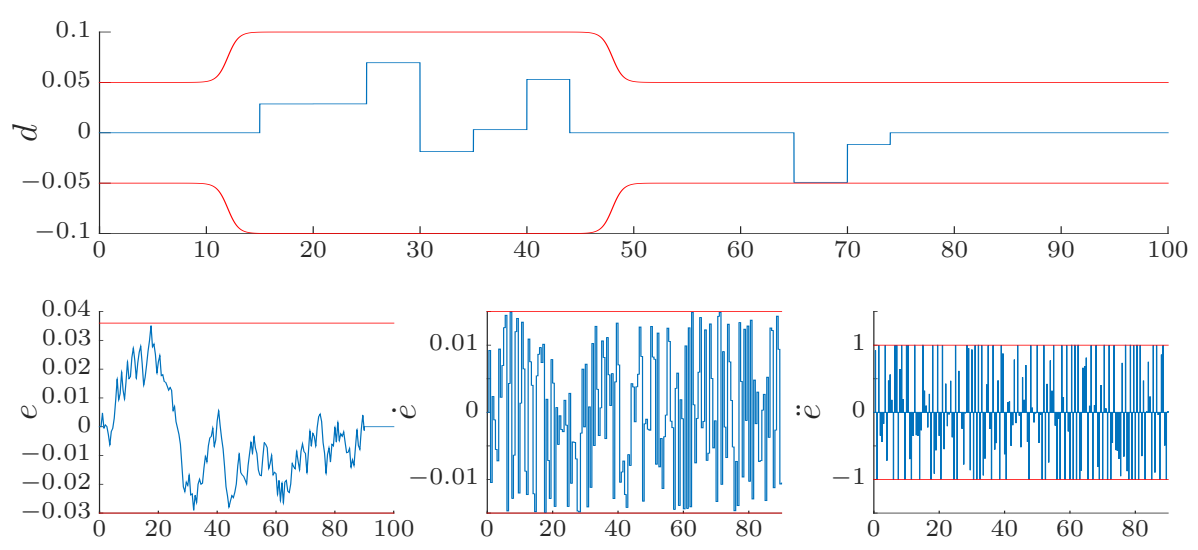

Figure 9. Disturbances $d$ and $e$ applied to the ball and beam system in simulation, with bounds in red.

\section{Example}

The ball and beam example which served as a case study in Sect. 2.2 is considered again for application of the method which was presented in Sect. 3 .

\section{$5.1 \quad$ Assumptions}

In this section, the assumptions in Sect. 2.4 are reviewed in the case of the ball and beam example introduced in Sect. 2.2.

- Assum. 1 and 2 are satisfied as was already illustrated on Fig. 7;

- As far as the relative degrees are concerned, $k=2$ and $l=2$ which fulfils Assum. 3 and 8;

- The disturbances and bounds are represented on Fig. 9. They fulfil Assum. 4;

- Assum. 5 is satisfied;

- The state-feedback controller with integral action proposed in Eq. (10) asymptotically stabilises the augmented system in Eq. (9). Also, it is strictly stable (see Eq. (11)), hence Assum. 6 is satisfied;

- The system is equivalent to a double-integrator with no transmission zero: $T_{u \rightarrow \alpha}(s)=-\frac{0.21}{s^{2}}$. Assum. 7 is fulfilled.

Remark 11: Note that the set-point $r_{s}=0.6 \mathrm{~m}$ (and $\dot{r}_{s}=0 \mathrm{~m} / \mathrm{s}$ ) is not the origin of the system (it is still a feasible equilibrium). However, using some transformation equivalent to a translation, one can obtain a set-point on the origin of the system. Hence Th. 2 and its proof are still valid.

\section{$5.2 \quad$ OIST implementation}

Using results in Sect. 3 and considering $\underline{\alpha}=0.1 \mathrm{~m}, \bar{\alpha}=0.9 \mathrm{~m}$, the following expressions are obtained for the successive $\Delta_{i}(t)$ :

$$
\forall t,\left\{\begin{array}{l}
\widehat{\Delta}_{0}(t)=0.8 \\
\widehat{\Delta}_{1}(t)=\kappa_{1}(t) \widehat{\lambda}_{1}^{d}(t)+\widehat{\lambda}_{1}^{n}(t) \\
\widehat{\Delta}_{2}(t)=\kappa_{2}(t) \widehat{\lambda}_{2}^{d}(t)+\widehat{\lambda}_{2}^{n}(t)
\end{array}\right.
$$


where

$$
\begin{aligned}
& \widehat{\lambda}_{1}^{d}(t)=\bar{\alpha}(t)-\underline{\alpha}(t)-2 g(\underline{e}(t), \bar{e}(t)) \\
& \widehat{\lambda}_{1}^{n}(t)=-2 g\left(\underline{e^{(1)}}(t), \overline{e^{(1)}}(t)\right) \\
& \widehat{\lambda}_{2}^{d}(t)=\left[\begin{array}{lll}
\kappa_{1}(t) & 1 & 0
\end{array}\right]\{\overline{\mathcal{A}}(t)-\underline{\mathcal{A}}(t)\}-2 f_{\text {abs }}\left(\left[\kappa_{1}(t) \quad 1 \quad 0\right]\right) g(\underline{\mathcal{E}}(t), \overline{\mathcal{E}}(t)) \\
& -2 g\left(\underline{e^{(1)}}(t), \overline{e^{(1)}}(t)\right) \\
& \widehat{\lambda}_{2}^{n}(t)=\left[\begin{array}{lll}
\dot{\kappa}_{1}(t) & \kappa_{1}(t) & 1
\end{array}\right]\{\overline{\mathcal{A}}(t)-\underline{\mathcal{A}}(t)\}-2 f_{\mathrm{abs}}\left(\left[\begin{array}{lll}
\dot{\kappa}_{1}(t) & \kappa_{1}(t) & 1
\end{array}\right]\right) g(\underline{\mathcal{E}}(t), \overline{\mathcal{E}}(t)) \\
& -2 g\left(\underline{e^{(2)}}(t), \overline{e^{(2)}}(t)\right)
\end{aligned}
$$

Then, the values of the design signals $\kappa_{i}(t)$ are deduced from these expressions and Th. 1:

$$
\begin{aligned}
& \kappa_{1}(t)=\frac{\breve{\kappa}_{1}-\lambda_{1}^{n}(t)}{\lambda_{1}^{d}(t)} \\
& \kappa_{2}(t)=\frac{\breve{\kappa}_{2}-\lambda_{2}^{n}(t)+2 \max \left(\left|\underline{e}^{(2)}(t)\right|,\left|\overline{e^{(2)}}(t)\right|\right)+2\left|\mathbf{C}_{\alpha} \mathbf{A B}_{d}\right| \max (|\underline{d}(t)|,|\bar{d}(t)|)}{\lambda_{2}^{d}(t)}
\end{aligned}
$$

where $\breve{\boldsymbol{\kappa}}=\left[\begin{array}{ll}\breve{\kappa}_{1} & \breve{\kappa}_{2}\end{array}\right]=\left[\begin{array}{ll}0.05 & 2\end{array}\right]$ are chosen so that the conditions in Th. 1 are satisfied. It is then possible to obtain saturations on the control signal in the form of Eq. (34). Note that $\mathbf{C}_{\alpha} \mathbf{A} \mathbf{B}_{u}=-0.21<0$ in this example so the operator in Eq. (27) is used to obtain the adequate saturations. As far as the anti-windup design is concerned, the time-varying coefficient $\boldsymbol{K}_{\text {oist }}(t)$ is defined as follows:

$$
\boldsymbol{K}_{\mathrm{oist}}(t)=\frac{\boldsymbol{U}^{2}(t) \Theta}{\mathbf{C}_{\alpha} \mathbf{A B}_{u}}
$$

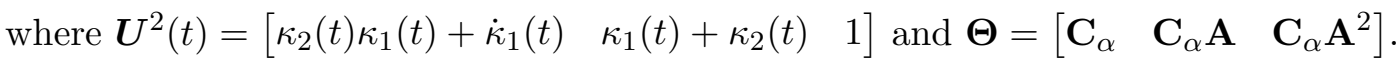

\subsection{Simulations and results}

Using the results in Sect. 5.2, simulations are performed over $100 \mathrm{~s}$. The disturbance signals used in simulation are shown in Fig. 9. At time $t=65 \mathrm{~s}$, the non-null disturbance input $d$ is used to show that the nominal control law is recovered when the time-domain constraint is not violated (see results). The simulation results are represented on Fig. 10 to 12. The data are represented in blue when considering the nominal control law only (no saturations) and in orange when the saturations obtained using OIST are introduced in the closed-loop. As mentioned in Sect. 2.2, the synthesized controller is not efficient enough and the ball falls off the beam. Using OIST and the knowledge on the disturbances bounds, the time-domain constraint is satisfied and nominal performance is recovered whenever the constraint is not violated. Note that the proposed approach results in some conservatism due to the lack of knowledge on $d$ and $e$, which do not necessarily equal their known bounds. Also, some conservatism is introduced by using differentiable upper-approximates of the absolute value and maximum functions.

On Fig. 11, it appears that the control law variations are much sharper in the saturated case. This is a trade-off required for complying with the time-domain requirement. Optimization of the constants $\breve{\boldsymbol{\kappa}}$ may help to obtain less demanding although satisfying control laws. This is considered future works. 

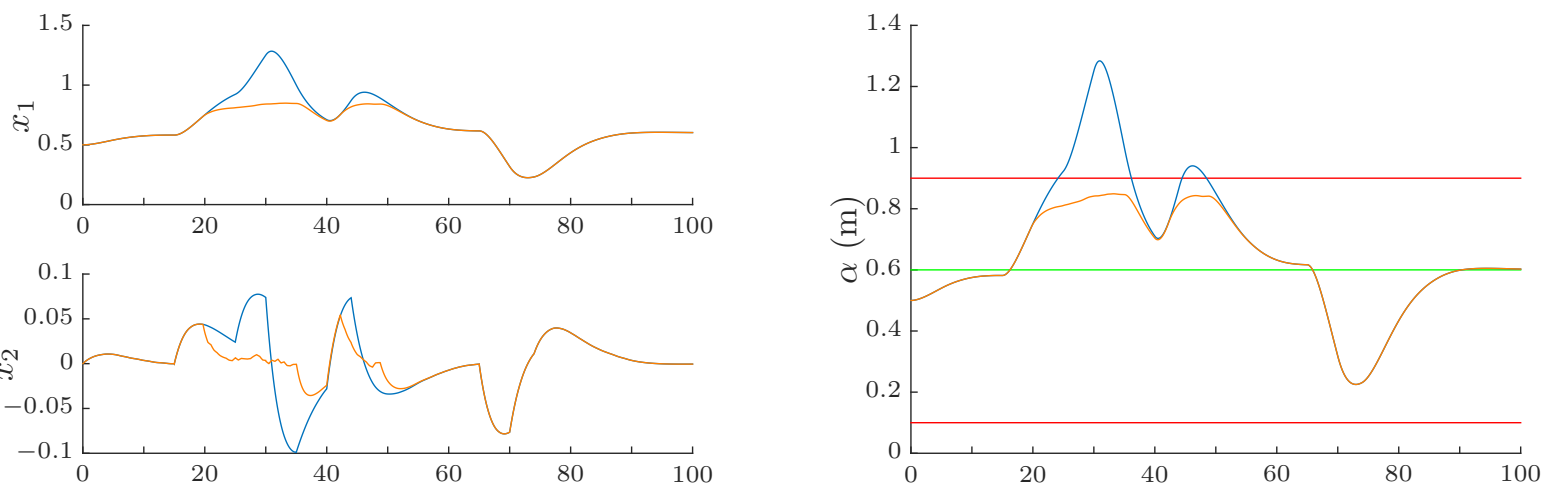

Figure 10. Left: in blue (resp. orange), unsaturated (resp. saturated) system state. Right: zoom on the regulated variable in the unsaturated (resp. saturated) case, in blue (resp. orange). The time-domain constraint on $\alpha$ is represented in red.
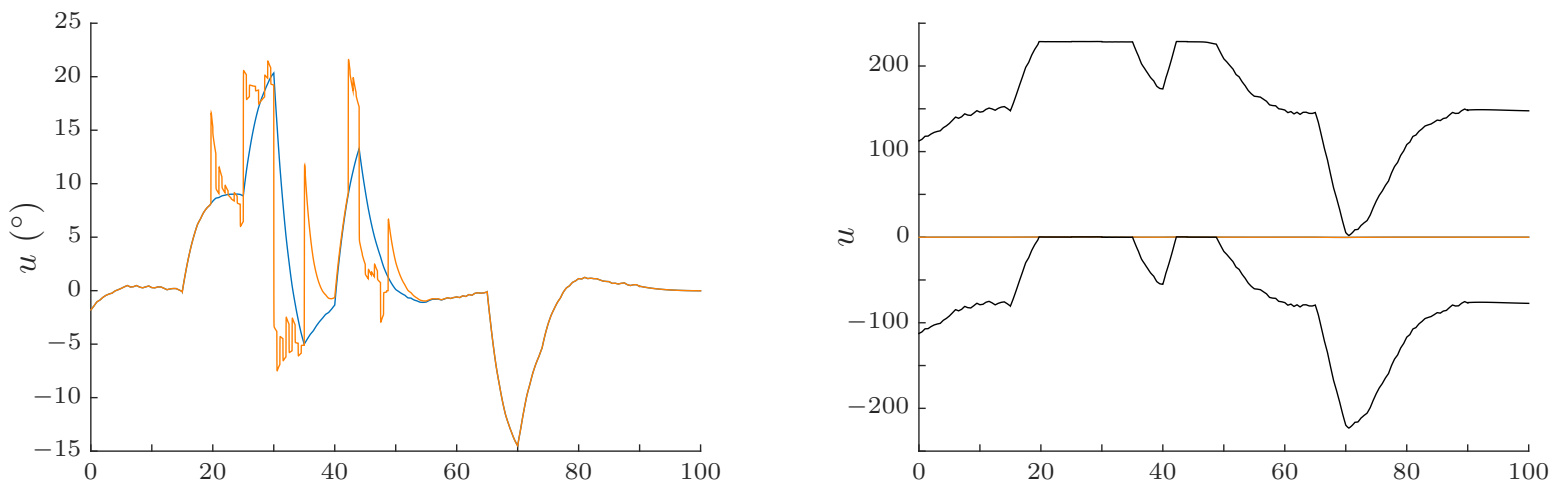

Figure 11. Left: in blue (resp. orange), unsaturated (resp. saturated) control signal. Right: the same control signal represented along with the saturations obtained using the OIST approach (in black).
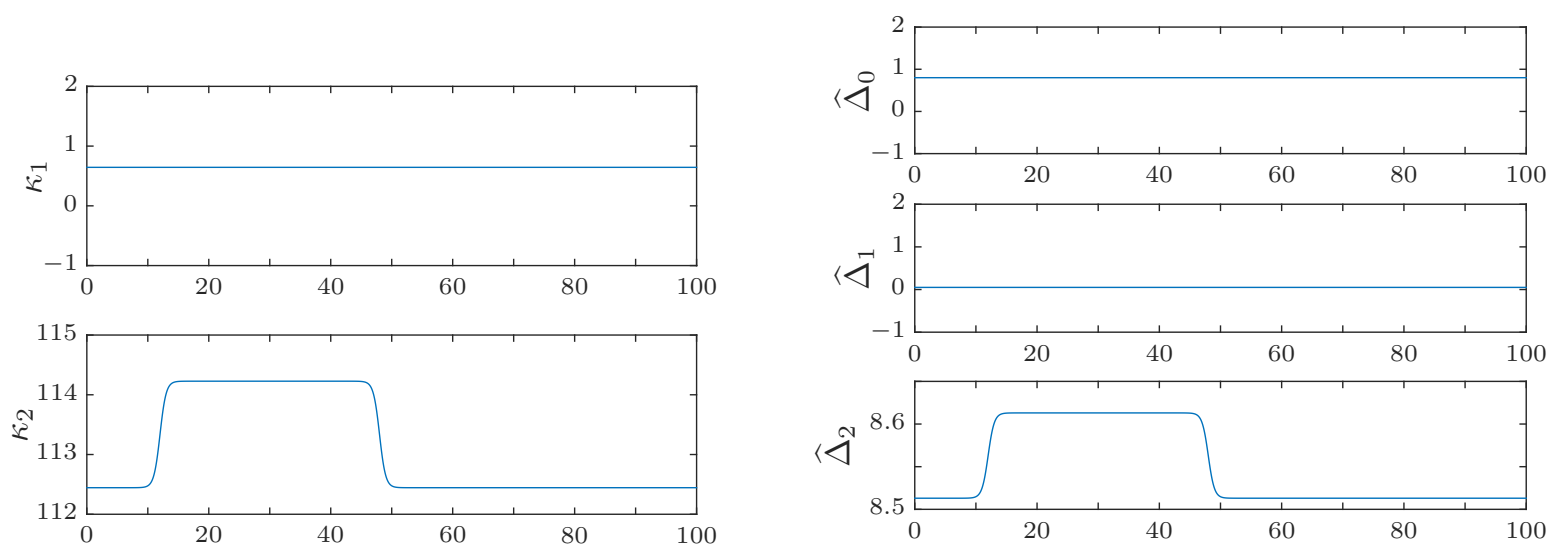

Figure 12. Left: time-varying coefficients $\kappa(t)$. Right: checking the saturations overlap.

On Fig. 12, the coefficients $\kappa_{1}(t)$ and $\kappa_{2}(t)$ as well as the quantities $\widehat{\Delta}_{i}(t)$ are represented. This allows to check that the assumptions and conditions in Th. 1 are fulfilled. 


\section{Conclusion}

In this article, the problem of keeping a linear system output - or regulated variable - in an interval has been formalized. A solution based on a transformation from the output expected "saturation" to a saturation on the existing linear control input has been proposed. A constructive method to apply this transformation has been introduced. Time-varying saturations are obtained and used in closed-loop. Special attention is paid to choose the OIST time-varying design parameters $\kappa_{i}(t)$ in Eq. (21) in order to avoid saturations overlap. Using results from the anti-windup design community, the stability of the system in closed-loop with the resulting non-linear control has been proved. An application to a linear ball and beam model has been proposed, showing satisfactory results.

However, throughout this article, the specific class of minimum-phase linear systems has been considered. Also, it has been supposed that the whole state is measured. Future works will be dedicated to extend the approach to non-minimum phase systems and to systems with output feedback. Optimizing the coefficients $\breve{\boldsymbol{\kappa}}$ will also be considered.

\section{References}

Apkarian, P., Ravanbod-Hosseini, L., \& Noll, D. (2011). Time domain constrained $\mathrm{H}_{\infty}$-synthesis. International Journal of Robust and Nonlinear Control, 21(2), 197-217.

Burlion, L. (2012). A new Saturation function to convert an output constraint into an input constraint. In Mediterranean Conference on Control and Automation (pp. 1218-1222). Barcelona, Spain.

Burlion, L., \& de Plinval, H. (2013). Keeping a Ground Point in the Camera Field of View of a Landing UAV. In Proceedings of the IEEE International Conference on Robotics and Automation (pp. 5763-5768).

Chambon, E., Burlion, L., \& Apkarian, P. (2015a). Output to input saturation transformation: Demonstration and application to disturbed linear systems. Osaka, Japan. (To appear in Proceedings of the 54th IEEE Conference on Decision and Control)

Chambon, E., Burlion, L., \& Apkarian, P. (2015b). Robust output interval constraint using O/I saturation transformation with application to uncertain linear launch vehicle. In Proceedings of the 14 th European Control Conference (pp. 1796-1801). Linz, Austria.

Chen, H., \& Allgöwer, F. (1996). A quasi-infinite horizon predictive control scheme for constrained nonlinear systems. In Proceedings of the 16th Chinese Control Conference (pp. 309-316). Qindao.

Chen, H., \& Allgöwer, F. (1999). Nonlinear model predictive control schemes with guaranteed stability. In R. Berber \& C. Kravaris (Eds.), Nonlinear model based process control. Dordrecht: Kluwer Academic.

Galeani, S., Tarbouriech, S., Turner, M., \& Zaccarian, L. (2009). A tutorial on modern anti-windup design. European Journal of Control, 15(3-4), 418-440.

Gevers, M. (2002). A decade of progress in iterative process control design: from theory to practice. Journal of Process Control, 12, 519-531.

Ghaemi, R., Sun, J., \& Kolmanovsky, I. (2012). Robust control of constrained linear systems with bounded disturbances. IEEE Transactions on Automatic Control, 57(10), 2683-2688.

Gilbert, E., \& Kolmanovsky, I. (2002). Nonlinear tracking control in the presence of state and control constraints: a generalized reference governor. Automatica, 38(12), 2063-2073.

Goodwin, G. C., Seron, M. M., \& de Doná, J. A. (2005). Constrained control and estimation. Springer-Verlag London.

Grimm, G., Hatfield, J., Postlethwaite, I., Teel, A. R., Turner, M. C., \& Zaccarian, L. (2003). Antiwindup for Stable Linear Systems with Input Saturation: An LMI-Based Synthesis. IEEE Transactions on Automatic Control, 48(9), 1509-1525.

Herrmann, G., Menon, P. P., Turner, M. C., Bates, D. G., \& Postlethwaite, I. (2010). Anti-windup synthesis for nonlinear dynamic inversion control schemes. International Journal of Robust and Nonlinear Control, 1465-1482.

Hu, X., Lindquist, A., Mari, J., \& Sand, J. (2012). Geometric Control Theory [Lecture notes]. KTH, Royal Institute of Technology, Sweden.

Isidori, A. (1999). Nonlinear control systems II. Springer London. 
Kapoor, N., \& Daoutidis, P. (1999). An observer-based anti-windup scheme for non-linear systems with input constraints. International Journal of Control, 72(1), 18-29.

Kapoor, N., Teel, A. R., \& Daoutidis, P. (1998). An Anti-Windup Design for Linear Systems with Input Saturation. Automatica, $34(5), 559-574$.

Khalil, H. K. (1996). Nonlinear Systems. New Jersey: Prentice Hall.

Menon, P. P., Herrmann, G., Turner, M. C., Bates, D. G., \& Postlethwaite, I. (2006). General Anti-windup synthesis for input constrained nonlinear systems controlled using nonlinear dynamic inversion. In Proceedings of the 45th IEEE Conference on Decision and Control (pp. 5435-5440). San Diego, CA, USA.

Mossberg, M., Gevers, M., \& Lequin, O. (2002). A comparison of iterative feedback tuning and classical PID tuning schemes. In Proceedings of the International Conference on Signal Processing, Robotics and Automation. Cadiz, Spain.

Polóni, T., Kalabić, U., McDonough, K., \& Kolmanovsky, I. (2014). Disturbance canceling control based on simple input observers with constraint enforcement for aerospace applications. In IEEE Conference on Control Applications (pp. 158-165).

Rojas, O. J., \& Goodwin, G. C. (2002). A simple anti-windup strategy for state constrained linear control. In Proceedings of the 15th IFAC World Congress. Barcelona, Spain.

Sontag, E. D. (1989). Remarks on stabilization and input-to-state stability. In Proceedings of the 28th IEEE Conference on Decision and Control (pp. 1376 - 1378). Tampa, FL: IEEE.

Tarbouriech, S., \& Turner, M. (2009). Anti-windup design: an overview of some recent advances and open problems. IET Control Theory and Applications, 3(1), 1-19.

Terrell, W. J. (2009). Stability and stabilization: an introduction. Princeton University Press.

Turner, M. C., \& Postlethwaite, I. (2002). Output violation compensation for systems with output constraints. IEEE Transactions on Automatic Control, 47(9), 1540-1546.

\section{Appendix A. Proof of Lemma 1}

The proof is performed iteratively with fixed $k$. Let $j$ such that $1 \leq j \leq k$. Suppose that $\alpha^{(k)}(t) \in$ $\left[\underline{\alpha^{(k)}}(t), \overline{\alpha^{(k)}}(t)\right] \Rightarrow \alpha^{(j)}(t) \in\left[\underline{\alpha^{(j)}}(t), \overline{\alpha^{(j)}}(t)\right], \forall t$. Also, $\alpha^{(j-1)}(0) \in\left[\underline{\alpha^{(j-1)}}(0), \overline{\alpha^{(j-1)}}(0)\right]$. Only the lower bound is considered. The demonstration is similar in the upper bound case. Suppose

$$
\exists t_{2}>0, \alpha^{(j-1)}\left(t_{2}\right)<\underline{\alpha^{(j-1)}}\left(t_{2}\right)
$$

then, since $\alpha^{(j-1)}(0) \in\left[\underline{\alpha^{(j-1)}}(0), \overline{\alpha^{(j-1)}}(0)\right]$ and, by continuity of $\alpha^{(j-1)}$ and $\underline{\alpha^{(j-1)}}$,

$$
\exists t_{1}, 0<t_{1}<t_{2},\left\{\begin{aligned}
\alpha^{(j-1)}\left(t_{1}\right) & =\underline{\alpha^{(j-1)}}\left(t_{1}\right) \\
\forall t \in\left[t_{1}, t_{2}\right], & \alpha^{(j-1)}(t) \leq \underline{\alpha^{(j-1)}}(t)
\end{aligned}\right.
$$

But, using the recurrence hypothesis, the definition of $\underline{\alpha^{(j)}}(t)$ and the fact that $\forall t, \kappa_{j}(t) \geq 0$, one obtains, $\forall t \in\left[t_{1}, t_{2}\right]$,

$$
\begin{aligned}
\alpha^{(j)}(t) & \geq \underline{\alpha^{(j)}}(t) \\
& \geq \kappa_{j}(t)\left(\underline{\alpha^{(j-1)}}(t)-\alpha^{(j-1)}(t)\right)+\overparen{\alpha^{(j-1)}}(t) \\
& \geq \overbrace{\alpha^{(j-1)}}(t)
\end{aligned}
$$


hence, using the property of integrals,

$$
\begin{aligned}
& \int_{t_{1}}^{t_{2}} \alpha^{(j)}(\lambda) \mathrm{d} \lambda \geq \int_{t_{1}}^{t_{2}} \overparen{\alpha^{(j-1)}}(\lambda) \mathrm{d} \lambda \\
& \alpha^{(j-1)}\left(t_{2}\right)-\alpha^{(j-1)}\left(t_{1}\right) \geq \underline{\underline{\alpha^{(j-1)}}}\left(t_{2}\right)-\underline{\alpha^{(j-1)}}\left(t_{1}\right)
\end{aligned}
$$

which contradicts Eq. (A1). In other words

$$
\forall t>0, \alpha^{(j-1)}(t) \geq \underline{\alpha^{(j-1)}}(t)
$$

which proves the lemma.

\section{Appendix B. Proof of Proposition 2}

Considering Eq. (42) and the system in Eq. (51), this problem is equivalent to studying the stability of the system $\dot{\boldsymbol{x}}(t)=\mathbf{A} \boldsymbol{x}(t)+\mathbf{B}_{u} u(t)$ in closed-loop with $v(t)=0$ or $u(t)=-\boldsymbol{K}_{\text {oist }}(t) \boldsymbol{x}(t)(d(t)=0$ and $e(t)=0)$. The transfer function between $u$ and the regulated variable $\alpha$ is given by

$$
\begin{aligned}
\alpha & =T_{u \rightarrow \alpha}(s) \\
& =\frac{s^{m}+p_{1} s^{m-1}+\ldots+p_{m-1} s+p_{m}}{s^{n}+d_{1} s^{n-1}+\ldots+d_{n-1} s+d_{n}} u
\end{aligned}
$$

with $k=n-m$ (see Assum. 3). Theoretically speaking, a minimum state-space representation of this transfer can be represented in the canonical form which can in turn be expressed as a chain of integrators in addition to the considered transfer zero dynamics, see (Hu, Lindquist, Mari, \& Sand, 2012, Chapter 4). The chain of integrators is given by

$$
\begin{cases}\dot{\alpha} & =\dot{\alpha} \\ & \vdots \\ \overparen{\alpha^{(k-2)}} & =\alpha^{(k-1)} \\ \overparen{\alpha^{(k-1)}} & =\alpha^{(k)} \\ & =-\boldsymbol{U}^{k}(t) \boldsymbol{\Theta} \boldsymbol{x}+\mathbf{C}_{\alpha} \mathbf{A}^{k} \boldsymbol{x}\end{cases}
$$

where the last equality is obtained by observing that $u=-K_{\text {oist }}(t) \boldsymbol{x}$ (and $d=0, e=0$ ). Let $\forall 0 \leq j \leq k-1, \gamma_{j}=\alpha^{(j)}+\boldsymbol{U}^{j}(t) \boldsymbol{\Theta} \boldsymbol{x}-\mathbf{C}_{\alpha} \mathbf{A}^{j} \boldsymbol{x}$ and $\boldsymbol{\Gamma}=\left[\begin{array}{ccc}\gamma_{0} & \cdots & \gamma_{k-1}\end{array}\right] \in \mathbb{R}^{k}$. Using Eq. (22) and (28) with null disturbances, the chain of integrators in Eq. (B2) can be re-written as 


$$
\begin{aligned}
\dot{\boldsymbol{\Gamma}} & =\left[\begin{array}{ccccc}
-\kappa_{1} & 1 & 0 & \ldots & 0 \\
0 & \ddots & \ddots & \ddots & \vdots \\
\vdots & \ddots & \ddots & \ddots & 0 \\
& & & -\kappa_{k-1} & 1 \\
0 & & \ldots & 0 & -\kappa_{k}
\end{array}\right] \boldsymbol{\Gamma} \\
& =\mathbf{A}_{\Gamma} \boldsymbol{\Gamma}
\end{aligned}
$$

This is completed by the zero dynamics as shown in (Hu et al., 2012, Chapter 4) which results in the open-loop transfer in Eq. (B1) being equivalent to the following state-space representation

$$
\overbrace{\left[\begin{array}{l}
\boldsymbol{\Gamma} \\
\boldsymbol{Z}
\end{array}\right]}^{\dot{2}}=\left[\begin{array}{cc}
\mathbf{A}_{\Gamma} & 0 \\
\mathbf{A}_{Z \Gamma} & \mathbf{A}_{Z}
\end{array}\right]\left[\begin{array}{l}
\boldsymbol{\Gamma} \\
\boldsymbol{Z}
\end{array}\right]
$$

where

$$
\mathbf{A}_{Z}=\left[\begin{array}{ccccc}
0 & 1 & 0 & \ldots & 0 \\
\vdots & \ddots & \ddots & \ddots & \vdots \\
\vdots & & \ddots & \ddots & 0 \\
0 & \ldots & \ldots & 0 & 1 \\
-p_{m} & -p_{m-1} & \ldots & -p_{2} & -p_{1}
\end{array}\right]
$$

and $\mathbf{A}_{Z \Gamma}$ is the null matrix except for the coefficient $\mathbf{A}_{Z \Gamma}(n-k, 1)=1$. Considering Assum. 7, $\mathbf{A}_{Z}$ eigenvalues are with strictly negative real parts. As far as the dynamics of Eq. (B4) is concerned, the following candidate Lyapunov positive definite function is considered

$$
V(\boldsymbol{\Gamma}, \boldsymbol{Z})=\frac{1}{2} \boldsymbol{\Gamma}^{\top} \boldsymbol{\Gamma}+\frac{\epsilon}{2} \boldsymbol{Z}^{\top} \boldsymbol{Z}
$$

where $\epsilon$ is a positive constant. Then

$$
\dot{V}(\boldsymbol{\Gamma}, \boldsymbol{Z})=\boldsymbol{\Gamma}^{\top} \mathbf{A}_{\Gamma} \boldsymbol{\Gamma}+\epsilon \boldsymbol{Z}^{\top} \mathbf{A}_{Z} \boldsymbol{Z}+\epsilon \boldsymbol{Z}^{\top} \mathbf{A}_{Z \Gamma} \boldsymbol{\Gamma}
$$

where, using the logarithmic function concavity and the fact that $\forall 1 \leq i \leq k, \gamma_{i-1} \gamma_{i} \leq\left|\gamma_{i-1} \gamma_{i}\right|$ :

$$
\begin{aligned}
\boldsymbol{\Gamma}^{\top} \mathbf{A}_{\Gamma} \boldsymbol{\Gamma} & =-\sum_{i=0}^{k-1} \kappa_{i+1} \gamma_{i}^{2}+\sum_{i=1}^{k-1} \gamma_{i-1} \gamma_{i} \\
& \leq-\sum_{i=0}^{k-1} \kappa_{i+1} \gamma_{i}^{2}+\frac{1}{2} \sum_{i=1}^{k-1} \gamma_{i}^{2}+\frac{1}{2} \sum_{i=0}^{k-2} \gamma_{i}^{2} \\
\boldsymbol{\Gamma}^{\top} \mathbf{A}_{\Gamma} \boldsymbol{\Gamma} & \leq-\boldsymbol{\Gamma}^{\top} \mathbf{D}_{V \Gamma} \boldsymbol{\Gamma} \\
& \leq-\boldsymbol{\Gamma}^{\top} \operatorname{diag}\left(\kappa_{1}-\frac{1}{2}, \kappa_{2}-1, \ldots, \kappa_{k-1}-1, \kappa_{k}-\frac{1}{2}\right) \boldsymbol{\Gamma}
\end{aligned}
$$

so that $\mathbf{D}_{V \Gamma}$ is a positive definite diagonal matrix upon adapted selection of the positive timevarying coefficients $\kappa_{i}(t)$. In the same vein 


$$
\epsilon \boldsymbol{Z}^{\top} \mathbf{A}_{Z \Gamma} \boldsymbol{\Gamma} \leq \frac{\epsilon \nu}{2}\left(\mathbf{A}_{Z \Gamma}^{\top} \boldsymbol{Z}\right)^{\top} \mathbf{A}_{Z \Gamma}^{\top} \boldsymbol{Z}+\frac{\epsilon}{2 \nu} \boldsymbol{\Gamma}^{\top} \boldsymbol{\Gamma}
$$

where $\nu$ is a positive constant. It comes that

$$
\dot{V}(\boldsymbol{\Gamma}, \boldsymbol{Z}) \leq-\boldsymbol{\Gamma}^{\top} \mathbf{D}_{V \Gamma} \boldsymbol{\Gamma}+\frac{\epsilon}{2 \nu} \boldsymbol{\Gamma}^{\top} \boldsymbol{\Gamma}+\epsilon\left(\boldsymbol{Z}^{\top} \mathbf{A}_{Z} \boldsymbol{Z}+\frac{\nu}{2} \boldsymbol{Z}^{\top}\left(\mathbf{A}_{Z \Gamma} \mathbf{A}_{Z \Gamma}^{\top}\right) \boldsymbol{Z}\right)
$$

Using the notations $\lambda_{\min }(\mathbf{M})$ and $\lambda_{\max }(\mathbf{M})$ to denote the minimal and maximal real parts of the eigenvalues of the matrix $M$, it is observed that

$$
\boldsymbol{Z}^{\top} \mathbf{A}_{Z} \boldsymbol{Z}+\frac{\nu}{2} \boldsymbol{Z}^{\top}\left(\mathbf{A}_{Z \Gamma} \mathbf{A}_{Z \Gamma}^{\top}\right) \boldsymbol{Z} \leq \lambda_{\max }\left(\mathbf{A}_{Z}\right) \boldsymbol{Z}^{\top} \boldsymbol{Z}+\frac{\nu}{2} \lambda_{\max }\left(\mathbf{A}_{Z \Gamma} \mathbf{A}_{Z \Gamma}^{\top}\right) \boldsymbol{Z}^{\top} \boldsymbol{Z}
$$

and

$$
-\boldsymbol{\Gamma}^{\top} \mathbf{D}_{V \Gamma} \boldsymbol{\Gamma}+\frac{\epsilon}{2 \nu} \boldsymbol{\Gamma}^{\top} \boldsymbol{\Gamma} \leq-\lambda_{\min }\left(\mathbf{D}_{V \Gamma}\right) \boldsymbol{\Gamma}^{\top} \boldsymbol{\Gamma}+\frac{\epsilon}{2 \nu} \boldsymbol{\Gamma}^{\top} \boldsymbol{\Gamma}
$$

By choosing $\nu=-\frac{\lambda_{\max }\left(\mathbf{A}_{Z}\right)}{\lambda_{\max }\left(\mathbf{A}_{Z \Gamma} \mathbf{A}_{Z \Gamma}^{\top}\right)}>0$ (since $\mathbf{A}_{Z \Gamma} \mathbf{A}_{Z \Gamma}^{\top}$ is positive semi-definite) and $\epsilon=$ $\nu \lambda_{\min }\left(\mathbf{D}_{V \Gamma}\right)>0$ and by observing that the eigenvalues of $\mathbf{A}_{Z}$ are with strictly negative real parts (see Assum. 7) and $\mathbf{D}_{V \Gamma}$ is a positive definite matrix, one obtains

$$
\begin{aligned}
\dot{V}(\boldsymbol{\Gamma}, \boldsymbol{Z}) & \leq-\frac{1}{2} \lambda_{\min }\left(\mathbf{D}_{V \Gamma}\right) \boldsymbol{\Gamma}^{\top} \boldsymbol{\Gamma}+\frac{1}{2} \lambda_{\max }\left(\mathbf{A}_{Z}\right) \boldsymbol{Z}^{\top} \boldsymbol{Z} \\
& \leq-\min \left(\lambda_{\min }\left(\mathbf{D}_{V \Gamma}\right),-\frac{1}{\epsilon} \lambda_{\max }\left(\mathbf{A}_{Z}\right)\right) V(\boldsymbol{\Gamma}, \boldsymbol{Z}) \\
& \leq-k_{1} V(\boldsymbol{\Gamma}, \boldsymbol{Z})
\end{aligned}
$$

where $k_{1}>0$. Also note that $\dot{V}(0,0)=0$. As a consequence, the candidate function $V$ is a Lyapunov function and the open-loop system $\dot{\boldsymbol{x}}=\left[\mathbf{A}-\mathbf{B}_{u} \boldsymbol{K}_{\text {oist }}(t)\right] \boldsymbol{x}$ is GES.

\section{Appendix C. Proof of Theorem 2}

First, considering Prop. 1, the origin is a reachable equilibrium of the system in closed-loop with the saturated control signal. Thus, it is of some interest to study the asymptotic stability of this equilibrium. Using Eq. (1), the state equation in Eq. (45) can be re-written as

$$
\dot{\boldsymbol{x}}(t)=\mathbf{A} \boldsymbol{x}(t)+\mathbf{B}_{u}\left[-\boldsymbol{K}_{\mathrm{oist}}(t) \boldsymbol{x}_{a}(t)+\mathbf{C}_{K} \boldsymbol{x}_{K}(t)+\mathbf{D}_{K}\left(\boldsymbol{y}(t)-\boldsymbol{x}_{a}(t)\right)-\mathrm{Dz}_{\underline{v}(t)}^{\bar{v}(t)}(v(t))\right]+\mathbf{B}_{d} d
$$

Using a similar approach to Kapoor and Daoutidis (1999), let define $\boldsymbol{e}_{x}(t):=\boldsymbol{x}(t)-\boldsymbol{x}_{a}(t)$. It follows that

$$
\begin{aligned}
& \dot{\boldsymbol{e}}_{x}(t)=\left(\mathbf{A}+\mathbf{B}_{u} \mathbf{D}_{K}\right) \boldsymbol{e}_{x}(t)+\mathbf{B}_{u} \mathbf{C}_{K} \boldsymbol{x}_{K}(t)+\mathbf{B}_{u} \mathbf{D}_{K} \mathbf{D}_{e} e(t)+\mathbf{B}_{d} d(t) \\
& \dot{\boldsymbol{x}}_{K}(t)=\mathbf{A}_{K} \boldsymbol{x}_{K}(t)+\mathbf{B}_{K} \boldsymbol{e}_{x}(t)+\mathbf{B}_{K} \mathbf{D}_{e} e(t)
\end{aligned}
$$


Let $\boldsymbol{X}=\left[\begin{array}{ll}\boldsymbol{e}_{x} & \boldsymbol{x}_{K}\end{array}\right]^{\top}$ and $\boldsymbol{W}=\left[\begin{array}{ll}d & e\end{array}\right]^{\top}$, then

$$
\begin{aligned}
\dot{\boldsymbol{X}} & =\left[\begin{array}{cc}
\mathbf{A}+\mathbf{B}_{u} \mathbf{D}_{K} & \mathbf{B}_{u} \mathbf{C}_{K} \\
\mathbf{B}_{K} & \mathbf{A}_{K}
\end{array}\right] \mathbf{X}+\left[\begin{array}{cc}
\mathbf{B}_{d} & \mathbf{B}_{u} \mathbf{D}_{K} \mathbf{D}_{e} \\
0 & \mathbf{B}_{K} \mathbf{D}_{e}
\end{array}\right]\left[\begin{array}{l}
d \\
e
\end{array}\right] \\
& =\mathbf{A}_{X} \boldsymbol{X}+\mathbf{B}_{X} \boldsymbol{W}
\end{aligned}
$$

Under Assum. 4 and $5,\|\boldsymbol{W}\|_{2}$ is finite and $\|\boldsymbol{W}\|$ is bounded. It follows that $\|\boldsymbol{X}\|_{2}$ is finite and $\|\boldsymbol{X}\|$ converges to zero. In case $\boldsymbol{W}=0$, the state $\boldsymbol{X}$ converges exponentially to zero. Replacing $v$ in Eq. (53) by its expression in Eq. (54), one obtains the following equation:

$$
\begin{aligned}
\dot{\boldsymbol{x}}_{a}(t)= & {\left[\mathbf{A}-\mathbf{B}_{u} \boldsymbol{K}_{\text {oist }}(t)\right] \boldsymbol{x}_{a}(t) } \\
& -\mathbf{B}_{u} \mathrm{Dz}_{\underline{v}(t)}^{\bar{v}(t)}\left(\boldsymbol{K}_{\text {oist }}(t) \boldsymbol{e}_{x}(t)+\boldsymbol{K}_{\text {oist }}(t) \mathbf{D}_{e} \boldsymbol{e}(t)+\mathbf{D}_{K} \boldsymbol{e}_{x}(t)+\mathbf{D}_{K} \mathbf{D}_{e} \boldsymbol{e}(t)+\mathbf{C}_{K} \boldsymbol{x}_{K}(t)\right)
\end{aligned}
$$

Considering Prop. 2, the open-loop system $\dot{\boldsymbol{x}}_{a}=\left[\mathbf{A}-\mathbf{B}_{u} \boldsymbol{K}_{\text {oist }}(t)\right] \boldsymbol{x}_{a}(t)$ is exponentially stable. Thus, for some positive definite function $V\left(\boldsymbol{x}_{a}\right)$, there exist by the converse Lyapunov theorem, see Khalil (1996), constants $\alpha_{i}, 1 \leq i \leq 4$, such that

$$
\begin{aligned}
\alpha_{1}\left\|\boldsymbol{x}_{a}\right\|^{2} & \leq V\left(\boldsymbol{x}_{a}\right) \leq \alpha_{2}\left\|\boldsymbol{x}_{a}\right\|^{2} \\
\left\|\frac{\partial V\left(\boldsymbol{x}_{a}\right)}{\partial \boldsymbol{x}_{a}}\right\| & \leq \alpha_{3}\left\|\boldsymbol{x}_{a}\right\| \\
\frac{\partial V\left(\boldsymbol{x}_{a}\right)}{\partial \boldsymbol{x}_{a}}\left[\mathbf{A} \boldsymbol{x}_{a}-\mathbf{B}_{u} \boldsymbol{K}_{\text {oist }}(t) \boldsymbol{x}_{a}\right] & \leq-\alpha_{4}\left\|\boldsymbol{x}_{a}\right\|^{2}
\end{aligned}
$$

Since, by Eq. (C4):

$$
\begin{aligned}
\dot{V}\left(\boldsymbol{x}_{a}\right) & =\frac{\partial V\left(\boldsymbol{x}_{a}\right)}{\partial \boldsymbol{x}_{a}}\left[\mathbf{A} \boldsymbol{x}_{a}-\mathbf{B}_{u} \boldsymbol{K}_{\text {oist }}(t) \boldsymbol{x}_{a}\right] \\
& -\frac{\partial V\left(\boldsymbol{x}_{a}\right)}{\partial \boldsymbol{x}_{a}} B_{u} \mathrm{Dz}_{\underline{v}(t)}^{\bar{v}(t)}\left(\boldsymbol{K}_{\text {oist }}(t) \boldsymbol{e}_{x}(t)+\mathbf{D}_{K} \boldsymbol{e}_{x}(t)+\mathbf{C}_{K} \boldsymbol{x}_{K}(t)+\left(\mathbf{D}_{K}+\boldsymbol{K}_{\text {oist }}(t)\right) \mathbf{D}_{e} e(t)\right)
\end{aligned}
$$

it comes

$$
\begin{aligned}
& \dot{V}\left(\boldsymbol{x}_{a}\right) \leq-\alpha_{4}\left\|\boldsymbol{x}_{a}\right\|^{2} \\
& +\alpha_{3}\left\|\boldsymbol{x}_{a}\right\|\left\|\mathbf{B}_{u}\right\|\left\|\mathrm{Dz}_{\underline{v}(t)}^{\bar{v}(t)}\left(\boldsymbol{K}_{\mathrm{oist}}(t) \boldsymbol{e}_{x}(t)+\mathbf{D}_{K} \boldsymbol{e}_{x}(t)+\mathbf{C}_{K} \boldsymbol{x}_{K}(t)+\left(\mathbf{D}_{K}+\boldsymbol{K}_{\mathrm{oist}}(t)\right) \mathbf{D}_{e} e(t)\right)\right\|
\end{aligned}
$$

First, using Lemma 3 and $\boldsymbol{e}_{x}:=\boldsymbol{x}-\boldsymbol{x}_{a}$, it is observed that since $\boldsymbol{K}_{\text {oist }}(t) \boldsymbol{x}$ is $K_{1}$-Lipschitz then

$$
\left\|\boldsymbol{K}_{\text {oist }}(t) \boldsymbol{e}_{x}\right\| \leq K_{1}\left\|\boldsymbol{e}_{x}\right\|, \forall t
$$

Second, by property of the deadzone function, $\left\|\mathrm{Dz}_{\underline{v}(t)}^{\bar{v}(t)}(v(t))\right\| \leq\|v(t)\|, \forall t$. It comes that 


$$
\begin{array}{r}
\dot{V}\left(\boldsymbol{x}_{a}\right) \leq-\alpha_{4}\left\|\boldsymbol{x}_{a}\right\|^{2}+\alpha_{3}\left\|B_{u}\right\|\left\|\boldsymbol{x}_{a}\right\|\left\{\left[K_{1}+\left\|\mathbf{D}_{K}\right\|\right]\left(\left\|\boldsymbol{e}_{x}\right\|+\left\|\mathbf{D}_{e}\right\|\|\boldsymbol{e}\|\right)+\left\|\mathbf{C}_{K}\right\|\left\|\boldsymbol{x}_{K}\right\|\right\} \\
\left.\leq-\alpha_{4}\left\|\boldsymbol{x}_{a}\right\|^{2}+\alpha_{3}\left\|B_{u}\right\|\left\|\boldsymbol{x}_{a}\right\|\left[\begin{array}{cc}
K_{1}+\|\left[\mathbf{D}_{K}\right. & \mathbf{C}_{K}
\end{array}\right] \|\right]\|\boldsymbol{X}\| \\
+\alpha_{3}\left\|B_{u}\right\|\left\|\boldsymbol{x}_{a}\right\|\left[K_{1}+\left\|\left[\begin{array}{ll}
0 & \mathbf{D}_{K}
\end{array}\right]\right\|\right]\left\|\mathbf{D}_{e}\right\|\|\boldsymbol{W}\|
\end{array}
$$

Using $k_{1}>0$ and $k_{2}>0$ such that

$$
\begin{aligned}
& k_{1} \geq \alpha_{3}\left\|\mathbf{B}_{u}\right\|\left[K_{1}+\left\|\left[\begin{array}{ll}
\mathbf{D}_{K} & \mathbf{C}_{K}
\end{array}\right]\right\|\right] \in \mathbb{R} \\
& k_{2} \geq \alpha_{3}\left\|\mathbf{B}_{u}\right\|\left[K_{1}+\left\|\left[\begin{array}{ll}
0 & \mathbf{D}_{K}
\end{array}\right]\right\|\right]\left\|\mathbf{D}_{e}\right\| \in \mathbb{R}
\end{aligned}
$$

then Eq. (C9) becomes

$$
\dot{V}\left(\boldsymbol{x}_{a}\right) \leq-\alpha_{4}\left\|\boldsymbol{x}_{a}\right\|^{2}+\left\|\boldsymbol{x}_{a}\right\|\left[k_{1}\|\boldsymbol{X}\|+k_{2}\|\boldsymbol{W}\|\right]
$$

Applying the inequality $2 \epsilon\left\|\boldsymbol{x}_{a}\right\|\|\boldsymbol{X}\| \leq \epsilon^{2}\left\|\boldsymbol{x}_{a}\right\|^{2}+\frac{\|\boldsymbol{X}\|^{2}}{\epsilon^{2}}$ for $\epsilon>0$, Eq. (C11) is re-written as

$$
\begin{aligned}
\dot{V}\left(\boldsymbol{x}_{a}\right) & \leq-\alpha_{4}\left\|\boldsymbol{x}_{a}\right\|^{2}+\frac{1}{2} k_{1}^{2} \epsilon_{1}^{2}\left\|\boldsymbol{x}_{a}\right\|^{2}+\frac{1}{2} k_{2}^{2} \epsilon_{2}^{2}\left\|\boldsymbol{x}_{a}\right\|^{2}+\frac{k_{1}^{2}}{2 \epsilon_{1}^{2}}\|\boldsymbol{X}\|^{2}+\frac{k_{2}^{2}}{2 \epsilon_{2}^{2}}\|\boldsymbol{W}\|^{2} \\
& \leq\left(-\alpha_{4}+\frac{1}{2} k_{1}^{2} \epsilon_{1}^{2}+\frac{1}{2} k_{2}^{2} \epsilon_{2}^{2}\right)\left\|\boldsymbol{x}_{a}\right\|^{2}+\frac{k_{1}^{2}}{2 \epsilon_{1}^{2}}\|\boldsymbol{X}\|^{2}+\frac{k_{2}^{2}}{2 \epsilon_{2}^{2}}\|\boldsymbol{W}\|^{2} \\
& \leq-\alpha_{5}\left\|\boldsymbol{x}_{a}\right\|^{2}+\frac{k_{1}^{2}}{2 \epsilon_{1}^{2}}\|\boldsymbol{X}\|^{2}+\frac{k_{2}^{2}}{2 \epsilon_{2}^{2}}\|\boldsymbol{W}\|^{2}
\end{aligned}
$$

where $\alpha_{5}=\alpha_{4}-\frac{1}{2} k_{1}^{2} \epsilon_{2}^{2}-\frac{1}{2} k_{2}^{2} \epsilon_{2}^{2}>0$ if the constants $\epsilon_{1}$ and $\epsilon_{2}$ are chosen small enough so that $\alpha_{4}>\frac{1}{2} k_{1}^{2} \epsilon_{1}^{2}+\frac{1}{2} k_{2}^{2} \epsilon_{2}^{2}$. Using (Isidori, 1999, Lemma 10.4.2, p.21), $V$ is thus an ISS-Lyapunov function for the system

$$
\dot{\boldsymbol{x}}_{a}=f_{1}\left(\boldsymbol{x}_{a},\left[\begin{array}{l}
\boldsymbol{X} \\
\boldsymbol{W}
\end{array}\right]\right)
$$

where $f_{1}$ is a non-linear function adequately defined. According to (Isidori, 1999, Theorem 10.4.1, p.21), the system in Eq. (C13) is thus ISS. At the beginning of this proof, it has been shown that for a specific class of bounded finite energy disturbances $d$ and $e-\|\boldsymbol{X}\|_{2}$ is finite and $\|\boldsymbol{X}\|$ converges to zero. Using a similar approach to the previous case, there exists $V_{X}$ and strictly positive constants $\beta_{1}, \beta_{2}$ such that $\dot{V}_{X}(\boldsymbol{X}) \leq-\beta_{1}\|\boldsymbol{X}\|^{2}+\beta_{2}\|\boldsymbol{W}\|^{2}$. This function is an ISS-Lyapunov function to the following system

$$
\dot{\boldsymbol{X}}=f_{2}(\boldsymbol{X}, \boldsymbol{W})
$$

where $f_{2}$ is a linear function adequately defined. Using (Isidori, 1999, Theorem 10.5.2, p.34), it is possible to conclude that the cascade of systems in Eq. (C15) is ISS. The cascade is illustrated on Fig. C1. Note that in case $d=0$ and $e=0$ and using (Isidori, 1999, Corollary 10.5.3, p.35), the origin $\left(\boldsymbol{x}_{a}, \boldsymbol{X}\right)=(0,0)$ is GAS for the cascade. 
